\title{
Application des dosages par immunonéphélémétrie microparticulaire des caséines $\alpha, \beta$ et $\kappa$ à l'évaluation de la qualité du lait, de sa production à sa valorisation fromagère
}

\author{
P Montagne ${ }^{1^{\star}}, \mathrm{ML}_{\text {Cuillière }}{ }^{1^{* \star}}, \mathrm{E} \mathrm{Marchal}^{1}, \mathrm{~N}$ El Bari ${ }^{1^{\star \star *}}$, \\ M Montagne ${ }^{1}$, M Benali ${ }^{1}$, G Faure ${ }^{1}$, J Duheille 1 , G Humbert ${ }^{2}$, \\ G Linden², N Heurtaux ${ }^{3}$, JL Blesche ${ }^{3}$, D Gosselin ${ }^{3}$, \\ A Desmares $^{3}$, D Delahaye ${ }^{3}$ \\ ${ }^{1}$ Laboratoire d'immunologie, faculté de médecine, BP 184, \\ F-54505 Vandouvre-lès-Nancy Cedex; \\ ${ }^{2}$ Laboratoire de biochimie appliquée associé à l'INRA, faculté des sciences, BP 239, \\ F-54506 Vandouvre-lès-Nancy Cedex; \\ ${ }^{3}$ UCL Isigny-Sainte-Mère, BP 93, F-14230 Isigny-sur-Mer, France
}

(Reçu le 6 octobre 1994 ; accepté le 27 février 1995)

Résumé - Les caséines $\alpha, \beta$ et $\kappa$ ont été dosées par immunonéphélémétrie microparticulaire, dans 1300 échantillons de lait, collectés bimensuellement pendant 13 mois (octobre 1992 à octobre 1993), auprès de 50 troupeaux de Normandie. Les concentrations de ces 3 caséines sont comparées à celles d'autres paramètres de composition, mesurés sur les mêmes laits (matières protéiques, matières grasses, lactose et extrait sec total). Les principaux facteurs, liés à l'exploitation, au troupeau, à sa conduite et à son alimentation, qui déterminent les paramètres de composition du lait à la production sont ensuite analysés. Ces mêmes paramètres de composition biochimique et des paramètres de coagulation à la présure (temps de floculation, indice de raffermissement $\mathrm{K} 15$, indice de fermeté $\mathrm{A} 30$ ) sont mesurés, sur la même période, dans des laits crus et standardisés en matière grasse, utilisés dans une fabrication fromagère industrielle. Réalisé dans un cadre de temps et d'espace limité, l'ensemble du travail envisage l'étude du lait depuis sa production jusqu'à sa valorisation par transformation fromagère. II permet de retrouver des notions bien connues et abondamment publiées, tant au niveau biochimique (liens entre les paramètres de composition, variation et variabilité de ceux-ci), que zootechnique (influence de la race, des potentialités pédologiques du sol, de la répartition des vêlages, de l'alimentation, des conditions

*INSERM ; "* CNRS ; ${ }^{\star \star \star}$ adresse actuelle : Laboratoire de microbiologie (M Ismaili), Faculté des sciences, BP 4010, Meknès, Maroc. 
de transition entre stabulation et pâturage) et technologique (influence relative des paramètres de composition sur la coagulabilité à la présure et les rendements de fabrication fromagère). L'immunonéphélémétrie microparticulaire, qui permet un dosage spécifique et précis des différentes caséines du lait, apparaît comme une technique originale d'immunoanalyse, complétant les moyens traditionnels d'évaluation de la qualité du lait.

caséine $\alpha$ / caséine $\beta$ / caséine $\kappa$ / immunonéphélémétrie microparticulaire / qualité du lait

Summary - Application of microparticle-enhanced nephelometric immunassays of $\alpha$-, $\beta$ - and $\mathrm{k}$-caseins to evaluate quality of milk, from the production to the valorization in cheese industry. $\alpha-, \beta$ - and $\kappa$-caseins were assayed, using a microparticle-enhanced nephelometric immunoassay, in 1300 milk samples, collected fortnightly during 13 months (october 1992 to october 1993), from 50 herds from Normandie (France). The concentration of these three caseins was compared with those of other milk compounds (protein, fat, lactose and dry matter). The main factors defining milk composition and related to the farm, herd, herd management and feeding, were then studied. The biochemical composition and rennet-coagulation (rennet coagulation time, firming time K15, and firmness parameter $\mathrm{A} 30$ ) were evaluated during the same period, in raw and fat-standardized milks, used in an industrial production of cheese. Performed in short and narrow limits, our work was a global study on milk from the production to the valorization in cheese industry. It allowed to confirm well known and copiously published results concerning milk biochemistry (relations between composition parameters, evolution and variability of these parameters), zootechnology (influence of breed, pedologic potentialities of the grazing soils, seasonal distribution of calving, feeding, conditions of transition from stabbling to pasture) and cheese-making (relative influence of the parameters of composition on the rennet-coagulability of milk and cheese-making yields). Microparticle-enhanced nephelometric immunoassay, allowing a specific and accurate assessment of the milk casein, thus appeared as an immunoanalytical method, completing the traditional tools for the evaluation of milk quality.

$\alpha$-casein / $\beta$-casein/ $\mathrm{k}$-casein /microparticle-enhanced nephelometric immunoassay/quality of milk

\section{INTRODUCTION}

La norme AFNOR $\times 50.109$ définit la qualité d'un produit comme son " aptitude à satisfaire les besoins des utilisateurs $n$ (Piat, 1986). En France et dans la plupart des grands pays laitiers, l'industrie fromagère, utilisatrice du produit lait, a été confrontée ces dernières années, à une diminution progressive de la qualité fromagère de la matière première qu'elle transforme. Cette diminution a abouti à une chute notable des rendements de fabrication et des qualités organoleptiques des produits fabriqués (Maubois, 1987).

L'intérêt des protéines dans la qualité du lait pour l'industriel et l'importance particulière de la fraction caséique en fabrication fromagère (Lenoir et Schneid, 1987) expliquent que l'estimation des richesses protéi- que et caséique du lait, des facteurs qui les déterminent à la production et de leur influence dans la transformation industrielle du lait en fromage, aient fait l'objet de nombreux travaux (Mahieu, 1985 ; Coulon, 1991 ; Hoden et al, 1991). Dans ces travaux, les teneurs du lait en protéines ou en caséine totale, qui peuvent être dosées par de nombreuses méthodes (Grappin, 1992 ; Grappin et Ribadeau-Dumas, 1992) sont le plus souvent retenues respectivement comme indice de la qualité protéique et caséique du lait. Les caséines $\alpha, \beta$ et $\kappa(\alpha-, \beta$ et $\kappa-\mathrm{CN}$ ), dont l'influence relative sur l'aptitude à la coagulation du lait et le rendement fromager demeure controversée (Colin et Laurent, 1991), sont plus rarement mesurées, peut-être parce que les moyens de leur détermination (électrophorèse, chromatographie), sont longtemps demeurés com- 
plexes, nécessitant souvent des étapes d'extraction, de concentration et de séparation (Guillou et al, 1987 ; Collin et al, 1991).

L'application des techniques immunologiques ouvre aujourd'hui de nouvelles perspectives analytiques dans le domaine de l'agro-alimentaire (Samarajeewa et al, 1991 ; Boquien, 1993). L'immunonéphélémétrie microparticulaire est une méthode d'immunoanalyse (Marchand et al, 1992 ; Montagne et al, 1992a) développée ces dernières années pour de nombreuses protéines sériques humaines (Montagne et al, $1992 b)$. Appliquée au lait et aux produits laitiers, elle permet actuellement le dosage spécifique des différentes $\mathrm{CN}$ (Collard-Bovy et al, 1991 ; El Bari et al, 1991 ; Humbert et al, 1991) et des principales protéines lactosériques (Marchal et al, 1991 ; Montagne et al, 1991).

L'immunonéphélémétrie microparticulaire est utilisée ici pour doser les $\alpha-\mathrm{CN}\left(\alpha_{\mathrm{S1}}\right.$ - et $\alpha_{s 2}-\mathrm{CN}$ ), $\beta-\mathrm{CN}$ et $\kappa-\mathrm{CN}$ au cours d'une étude globale du lait depuis sa production jusqu'à sa valorisation par transformation fromagère. Cette étude, réalisée en Normandie, s'étend sur 52 semaines réparties sur 13 mois, d'octobre 1992 à octobre 1993. Les concentrations des $3 \mathrm{CN}$ et celles d'autres paramètres de composition tels que taux protéique (TP), taux butyreux (TB), lactose et extrait sec total (EST), mesurées sur les mêmes échantillons par les méthodes traditionnellement utilisées pour évaluer la qualité du lait, sont étudiées parallèlement dans 3 approches complémentaires : i) étude des paramètres de composition biochimique de laits de troupeau dans des conditions normales de production, de leur évolution saisonnière et des liens statistiques qui existent entre eux ; ii) influence, sur les paramètres de composition précédemment définis, de divers facteurs liés à l'exploitation (potentialités pédologiques des sols), au troupeau (race dominante), à sa conduite (répartition saisonnière des vêlages) et à son alimenta- tion (aspects quantitatif et qualitatif de l'alimentation, conduite des transitions entre stabulation et pâturage) ; iii) étude de laits de grand mélange utilisés dans une fabrication fromagère industrielle et influence, au niveau technologique de la transformation du lait en fromage (coagulation à la présure, rendements de fabrication), des paramètres précédemment étudiés sur les laits de troupeau.

L'objectif de ce travail a été d'évaluer l'intérêt de l'analyse spécifique des $\alpha$-, $\beta$ - et $\kappa$ $\mathrm{CN}$ dans l'étude biochimique du lait, depuis sa production jusqu'à sa valorisation par transformation fromagère, afin d'apprécier ce qu'une telle méthode immunochimique peut apporter à l'ensemble des acteurs de la filière lait.

\section{MATÉRIEL ET MÉTHODES}

\section{Troupeaux et laits de troupeau}

\section{Caractéristiques des troupeaux}

L'étude des laits de troupeau porte sur 50 troupeaux sélectionnés, en fonction des résultats du contrôle laitier, sur 3 critères vérifiés sur les 7 mois précédant son début : la qualité bactériologique (tous les laits étaient classés $A_{\text {, }}<100000$ germes $/ \mathrm{ml}$ ou super $A_{1}<50000$ germes $/ \mathrm{ml}$ ), le TP du lait et la race dominante $(\geq 85 \%)$ du troupeau. La répartition des troupeaux selon ces 2 derniers critères est la suivante : le TP du lait était $<32,5 \mathrm{~g} / \mathrm{l}$, compris entre 32,5 et $33,5 \mathrm{~g} / \mathrm{l}$ et $>33,5$ g/l, pour 16,17 et 17 troupeaux respectivement; la race Normande prédominait dans 19 troupeaux, la race Française Frisonne Pie Noire (FFPN) dans 13 et les 18 autres étaient des troupeaux mixtes Normande - FFPN.

La répartition géographique des troupeaux étudiés est étroite : 49 d'entre eux sont localisés dans le département de la Manche autour de Sainte-Mère-Église dans un cercle d'environ $20 \mathrm{~km}$ de diamètre grossièrement limité par Carentan, Saint-Sauveur-le-Vicomte et Valognes. Quarante-six sont en zone d'Appellation d'origine contrôlée " crème et beurre d'Isigny-surMer ". Le nombre total de vaches par troupeau varie entre 8 et 115 (moyenne: 45 ), la surface ex- 
ploitée par le producteur, de 11 à 155 ha (moyenne, $58 \mathrm{ha}$ ) et les quotas s'échelonnent de 24000 à 611000 I par an (moyenne : 192660 I).

Au cours de l'étude, quelques changements affectant le groupe sélectionné imposent quelquefois d'exclure certains troupeaux des calculs statistiques. Ces changements concernent principalement un troupeau sorti de l'étude en décembre 1992 et le changement de la race dominante dans 2 autres troupeaux (Normande à troupeau mixte, juillet 1993 et troupeau mixte à Normande, août 1993).

\section{Échantillonnage des laits de troupeau}

L'échantillonnage des laits de chaque troupeau, 1300 échantillons au total sur les 13 mois de l'étude, a été réalisé à la ferme 2 fois par mois. Les prélèvements ont tous été effectués par une même personne en dehors des tournées de ramassage normales, sur tanks ( 2 à 4 traites) réfrigérés et maintenus sous agitation. Les échantillons $(30 \mathrm{ml})$, additionnés d'azide de sodium à $0,2 \%$ et strictement conservés entre 1 et $4^{\circ} \mathrm{C}$ (contrôle par sonde thermique), ont été répartis entre les différents sites d'analyse. Les dosages ont été effectués dans les $72 \mathrm{~h}$ qui suivent le prélèvement.

\section{Informations recueillies sur les troupeaux}

Plus de 25000 informations ont été collectées auprès des 50 producteurs sélectionnés, à l'aide d'un questionnaire rigide prédéfini, rempli par la personne assurant la collecte des échantillons au cours d'entretiens bimensuels avec les producteurs. Ces données concernent l'exploitation (surface, localisation, géologie, pédologie), le troupeau (nombre de vaches en lactation, de vêlages, de vaches taries, de cas de mammites, stabulation ou pâturage), son alimentation (nature et quantité d'aliments par vache et par jour) et le lait produit (volume tank $24 \mathrm{~h}$ ). Les données concernant les potentialités pédologiques des sols et l'alimentation des troupeaux sont détaillées dans les paragraphes suivants.

\section{Potentialités pédologiques des sols}

L'étude de linfluence du sol sur la qualité du lait produit est limitée à la recherche de liens de dé- pendance entre les principaux paramètres de composition mesurés sur les laits et des facteurs géo-pédologiques simples déterminant la qualité potentielle des herbages. Elle ne constitue donc qu'une approche très restrictive du terroir puisqu'elle n'inclue aucune notion zoologique, historique ou humaine (Laville, 1993 ; Linden et Chamba, 1994).

Les sols sont inventoriés dans la région d'élevage des troupeaux étudiés et leur potentialité à produire des herbages de qualité est estimée en fonction de caractéristiques liées à leur nature (Duchaufour, 1970) à celle de leur substratum géologique (Goguel, 1967) et à l'histoire géo-pédologique locale (Doré et al, 1977 ; Desnouveaux, 1984). Un sol est d'autant plus favorable à un herbage de qualité que sa teneur en calcium le rend proche de la neutralité et qu'il n'est ni trop sec, ni gorgé d'eau (Duchaufour, 1970). La classification des sols inventoriés selon leur acidité et leur humidité est donc utilisée pour déterminer ceux qui sont très favorables, favorables, et peu favorables à un herbage de bonne qualité. La nomenclature utilisée est celle de la classification française des sols (CPCS, 1967).

\section{Alimentation des troupeaux}

La ration alimentaire journalière ( $\mathrm{kg} / \mathrm{vache} / \mathrm{jour}) \mathrm{u}$ tilisée dans l'étude de l'aspect quantitatif de l'alimentation correspond à la somme des divers aliments donnés aux troupeaux. Elle n'inclut aucune information concernant la valeur énergétique ou nutritionnelle de ces aliments.

L'étude de l'aspect qualitatif de lalimentation nécessite une synthèse préalable de la masse d'informations collectées comme indiqué précédemment. Une alimentation de base et une alimentation complémentaire sont ainsi définies. L'alimentation de base regroupe les aliments les plus importants quantitativement (herbe, ensilage de maïs, ensilage d'herbe, foin). Selon les troupeaux, elle peut être constituée d'un aliment principal, constant au pâturage ou en stabulation, et d'aliments secondaires inconstants, donnés en alternance ou en mélange avec d'autres. L'alimentation complémentaire concerne des aliments foumis aux troupeaux en plus faible quantité (betteraves, soja, blé, orge, avoine, luzerne, aliment concentré de composition inconnue). Elle est constituée d'aliments donnés régulièrement sur les 13 mois de l'étude, ou d'aliments donnés irrégulièrement, mais assurant quelquefois avec 
d'autres une alimentation complémentaire constante.

La transition stabulation-pâturage est définie comme la période pendant laquelle le troupeau, tout en étant encore en stabulation la nuit, consomme déjà de l'herbe au cours de la journée.

\section{Laits de fromagerie et fabrications fromagères}

L'étude des laits de fromagerie a porté sur 178 échantillons : 89 laits crus de grand mélange (environ 100000 I) réceptionnés par la laiterie qui collecte les laits des 50 troupeaux étudiés précédemment, et les mêmes laits standardisés en MG (environ $25 \mathrm{~g} / \mathrm{l}$ ). Les prèlèvements ont été effectués sur les tanks réfrigérés et maintenus sous agitation et les échantillons ont été traités comme ceux des laits de troupeau.

Les 65 fabrications fromagères étudiées, de type pâte pressée demi-cuite (FIL, 1981), ont été effectuées par coagulation ( $30 \mathrm{~min}, \mathrm{pH}$ standardisé, température appropriée, chymosine animale, ferments, $\mathrm{CaCl}_{2}$ ) des laits précédents après standardisation en MG et bactofugation. L'échantillonnage a été effectué à la sonde sur un bloc de caillé en position repérée et constante dans le bac de placage-découpage. Le volume du lait entrant en fabrication fromagère, le poids total et les matières azotées totales (MAT) du fromage obtenu en sortie de saumure, le nombre de pièces fabriquées et le poids moyen d'une pièce ont été mesurés sur chaque fabrication. Les rendements de fabrication sont exprimés en $\mathrm{kg}$ de fromage (rendement brut) et en $\mathrm{kg}$ de MAT (rendement MAT) obtenus à partir de 1001 de lait standardisé en MG entrant en fabrication.

\section{Analyses réalisées sur les laits}

Plus de 13500 analyses ont été effectuées sur l'ensemble des laits de troupeau et des laits de fromagerie étudiés. Les concentrations en $\alpha-\mathrm{CN}$, $\beta-C N$ et $\kappa-C N$ sont mesurées 2 fois par mois sur les laits de troupeau par immunonéphélémétrie microparticulaire. Les TP, TB, teneur en lactose et I'EST des mêmes échantillons sont dosés par absorption dans l'infra-rouge (Biggs et al, 1987 ; FIL, 1990), dans un laboratoire interprofessionnel agrée de contrôle laitier local. Pour chaque lait de fromagerie cru et chaque lait standardisé en MG, les paramètres de coagulation (temps de flocula- tion, indice de raffermissement $\mathrm{K} 15$, indice de fermeté $\mathrm{A} 30$ ) et la teneur en calcium total (calcimètre Corning 940, Corning Inc, États-Unis) sont mesurés en plus des paramètres de composition.

\section{Dosage des $\alpha-C N, \beta-C N$ et $\kappa-C N$ par immunonéphélémétrie microparticulaire}

Les concentrations des laits en $\alpha-\mathrm{CN}, \beta-\mathrm{CN}$ et $\kappa$ $\mathrm{CN}$ ont été déterminées par immunonéphélémétrie microparticulaire, en inhibition par l'analyte $\alpha$ $(\beta, \kappa)$ - $\mathrm{CN}$ de l'agglutination d'un conjugué microparticule- $\alpha(\beta, \kappa)-C N$, obtenue en présence d'un antisérum de lapin anti- $\alpha(\beta, \kappa)$ - $\mathrm{CN}$ bovine (Collard-Bovy et al, 1991 ; El Bari et al, 1991). Après chauffage du lait entier à $40^{\circ} \mathrm{C}$ et homogénéisation, l'échantillon est prédilué au $1 / 3500$ ( $1 / 50$ puis $1 / 70)$. Le lait prédilué ou le standard ou le contrôle $(30 \mu l)$ est mélangé dans une cuve de mesure néphélémétrique (Néphélia microcuvette, volume réactionnel $300 \mu \mathrm{l}$, Sanofi Diagnostics Pasteur, Marne-la-Coquette, France) avec le conjugué $(30 \mu \mathrm{l})$ et la prédilution de travail de l'antisérum $(30 \mu l)$. Toutes les prédilutions et les mises en réaction ont été effectuées à l'aide d'un diluteur (Microlab 900, Hamilton, Bonaduz, Suisse) dans le tampon pour néphélémétrie décrit par CollardBovy et al (1991). Les mesures néphélémétriques (Néphélémètre Néphélia N600, Sanofi Diagnostics Pasteur) ont été réalisées après $1 \mathrm{~h}$ d'incubation au repos à température ambiante. Les concentrations des échantillons ont été calculées par rapport à une courbe étalon réalisée dans les mêmes conditions que les essais à l'aide d'une poudre de lait préalablement standardisée (Collard-Bovy et al, 1991). Des contrôles, placés en début et en fin de série, ont été réalisés pour chaque dosage et permettent d'évaluer les répétabilités intra-et inter-essai sur les 13 mois de l'étude.

\section{Mesure des paramètres de la coagulation}

Une détermination rhéologique de la cinétique de coagulation et de la texture du gel après emprésurage a été réalisée pour chaque lait de fromagerie, cru et standardisé. Le temps de floculation (temps écoulé entre l'emprésurage et le début de la coagulation) a été déterminé (Sommer et Matsen, 1935) par l'observation d'un mince film de lait s'écoulant sur la paroi d'un tube de verre incliné, 
tournant lentement dans un bain d'eau à $35^{\circ} \mathrm{C}$ et contenant $10 \mathrm{ml}$ de lait et $500 \mu \mathrm{l}$ de solution de présure (HALA X, 0,25\% en phosphate $50 \mathrm{mmol} / \mathrm{l}, \mathrm{pH} 6,5)$. L'évolution des propriétés mécaniques du gel (Tarodo de la Fuente et al, 1969), obtenu à $35^{\circ} \mathrm{C}$ sur $350 \mu \mathrm{l}$ du mélange précédent, est suivie pendant $40 \mathrm{~min}$ à laide d'un thrombélastographe (Thrombocord, LARA SA, Chevigny, France). Le profil de coagulation tracé par l'appareil permet la mesure de l'indice de raffermissement K15 (temps écoulé, en min, après le début de la coagulation pour obtenir une fermeté du gel correspondant à une amplitude du tracé de $15 \mathrm{~mm}$ ) et la mesure de l'indice de fermeté A30 (amplitude du tracé, en $\mathrm{mm}, 30 \mathrm{~min}$ après emprésurage).

\section{Analyse statistique des résultats}

Les données mesurées sur l'ensemble des laits de troupeau et des laits de fromagerie, ou calculées (somme $\alpha-+\beta-+\kappa-C N$, rapport somme $\alpha$ $+\beta-+\kappa-C N / T P$, moyennes mensuelles et annuelles), ont été soumises à une analyse statistique classique (Foucart, 1984 ; Wonnacott et Wonnacott, 1992). L'analyse en régression linéaire de seconde espèce a été utilisée pour rechercher toutes les corrélations susceptibles d'exister entre les paramètres considérés 2 à 2. La signification contre 0 des coefficients de corrélation simple obtenus est évaluée par le test de Student à $(n-2)$ degrés de liberté. Les comparaisons des moyennes sont effectuées par le test de l'écart t de Student à $\left(n_{1}+n_{2}-2\right)$ degrés de liberté.

Pour chaque paramètre de composition mesuré ou calculé sur les laits de troupeau, les indices moyens et individuels de variation sont déterminés selon la méthode du rapport à la moyenne mobile centrée décrite par Wonnacott et Wonnacott (1992) : le calcul des moyennes mobiles est effectué par moyenne de 4 résultats successifs, pas de 1 et recouvrement; les moyennes mobiles centrées sont ensuite obtenues par moyenne des moyennes mobiles précédentes, considérées 2 à 2 ; puis l'indice moyen de variation (et les indices de variation individuels) sont calculés pour chaque mois, chaque paramètre (et chaque troupeau) par le rapport des valeurs mesurées aux moyennes mobiles centrées. Les indices moyens de variation de la somme $\alpha-+\beta-+\kappa-C N$, calculés ainsi sur l'ensemble des laits de troupeau, ont été utilisés pour estimer, dans le lait de chaque troupeau, les variations de la somme $\alpha-+\beta-+\kappa-C N$ corrigées des variations moyennes (rapport mensuel de la valeur mesurée sur l'indice moyen de variation). Les pourcentages de variation individuelle non expliquée par les variations moyennes sont finalement évalués à l'aide du rapport de l'indice moyen de variation sur l'indice de variation individuel. Ces pourcentages de variation de la somme $\alpha-+\beta-+\kappa-C N$ non expliquée par les variations moyennes sont analysées, cas par cas, en relation avec les informations collectées sur l'exploitation, le troupeau, sa conduite et son alimentation. Cette analyse permet de définir les facteurs qui semblent déterminer, à la production. les variations de la somme $\alpha-+\beta-+\kappa-C N$ du lait de chacun des troupeaux. Une synthèse comparative des facteurs ainsi définis et l'étude de leur influence sur chacun des autres paramètres de composition traditionnellement mesurés sur les laits, a été effectuée pour l'ensemble des 50 troupeaux. Les résultats de cette synthèse ont été finalement réévalués troupeau par troupeau.

\section{RÉSULTATS}

\section{Paramètres de composition des laits de troupeau}

\section{Étude globale des paramètres}

L'immunonéphélémétrie microparticulaire permet le dosage des $\alpha-\mathrm{CN}, \beta-\mathrm{CN}$ et $\kappa-\mathrm{CN}$ dans les 1300 échantillons de lait de troupeau avec des domaines de mesure respectifs de 3,3 à 52,5 , de 2,1 à 32,8 et de 0,8 à $12,6 \mathrm{~g} / \mathrm{l}$. Les études de répétabilité effectuées sur les contrôles indiquent des coefficients de variation (CV) moyens intra-essai de $2,9,3,0$ et $3,3 \%$ pour respectivement $\alpha$ $C N, \beta-C N$ et $\kappa-C N$. Pour les mêmes dosages, les CV inter-essai $(n=208)$ sont égaux respectivement à $4,4,3,7$ et $7,0 \%$.

Le tableau I indique pour chaque paramètre de composition mesuré sur les laits, les valeurs minimales, maximales et moyennes obtenues sur l'ensemble des troupeaux et selon leur type (race Normande dominante, mixte Normande-FFPN et race FFPN dominante). Pour l'ensemble des 50 troupeaux, le rapport de la somme $\alpha-+\beta-+\kappa-C N$ sur le TP 
Tableau I. Valeurs minimales, maximales et moyennes des paramètres de composition mesurés sur les laits de troupeau.

General statistics of milk parameters.

\begin{tabular}{|c|c|c|c|c|c|c|c|}
\hline $\begin{array}{l}T P \\
(g / l)\end{array}$ & $\begin{array}{l}T B \\
(g / l)\end{array}$ & $\begin{array}{l}\text { Lactose } \\
(\mathrm{g} / \mathrm{l})\end{array}$ & $\begin{array}{l}E S T \\
(g / l)\end{array}$ & $\begin{array}{l}\alpha-C N \\
(g / l)\end{array}$ & $\begin{array}{l}\beta-C N \\
(g / l)\end{array}$ & $\begin{array}{l}\kappa-C N \\
(g / l)\end{array}$ & $\begin{array}{l}C N^{1} \\
(g / l)\end{array}$ \\
\hline
\end{tabular}

18 troupeaux à race Normande dominante

$\begin{array}{lrrrrrrrr}\text { Moy } & 33,5 & 43,3 & 49,3 & 133,7 & 10,8 & 6,8 & 2,7 & 20,3 \\ \mathrm{ET}^{2} & 1,2 & 3,4 & 0,7 & 4,6 & 0,9 & 0,5 & 0,2 & 1,6 \\ \text { Min } & 31,7 & 38,3 & 48,0 & 127,9 & 9,6 & 6,0 & 2,4 & 18,0 \\ \text { Max } & 36,6 & 53,5 & 51,1 & 148,3 & 13,3 & 8,2 & 3,2 & 24,7\end{array}$

19 troupeaux mixtes Normande-FFPN

$\begin{array}{lrrrrrrrr}\text { Moy } & 32,8 & 43,1 & 49,1 & 132,5 & 10,3 & 6,5 & 2,6 & 19,4 \\ \text { ET } & 1,0 & 1,6 & 0,7 & 2,7 & 0,6 & 0,4 & 0,2 & 1,1 \\ \text { Min } & 30,3 & 40,6 & 47,9 & 127,8 & 8,9 & 5,6 & 2,2 & 16,8 \\ \text { Max } & 34,2 & 45,9 & 50,7 & 138,1 & 11,5 & 7,2 & 3,0 & 21,6\end{array}$

12 troupeaux à race FFPN dominante

$\begin{array}{lrrrrrrrr}\text { Moy } & 31,9 & 41,7 & 49,0 & 130,4 & 10,0 & 6,2 & 2,5 & 18,7 \\ \text { ET } & 0,5 & 1,5 & 0,5 & 2,1 & 0,3 & 0,2 & 0,1 & 0,5 \\ \text { Min } & 31,3 & 38,6 & 48,2 & 126,4 & 9,4 & 5,9 & 2,4 & 17,7 \\ \text { Max } & 35,3 & 47,9 & 49,9 & 140,6 & 10,4 & 6,5 & 2,7 & 19,4\end{array}$

Ensemble des 50 troupeaux

\begin{tabular}{lrrrrrrrr} 
Moy & 32,9 & 42,9 & 49,1 & 132,4 & 10,4 & 6,5 & 2,6 & 19,6 \\
ET & 1,7 & 2,9 & 1,0 & 4,4 & 1,0 & 0,6 & 0,3 & 1,7 \\
\hline
\end{tabular}

${ }^{1} \mathrm{CN}$ : somme $\alpha-+\beta-+\kappa-\mathrm{CN} ;{ }^{2} \mathrm{ET}$ : écart type.

est faible $(60 \%)$ et les proportions des différentes fractions caséiniques dans leur somme atteignent 53,33 et $13 \%$, respectivement pour $\alpha-\mathrm{CN}, \beta-\mathrm{CN}$ et $\kappa-\mathrm{CN}$. Ces proportions sont très constantes d'un troupeau à l'autre, quelle que soit sa race dominante.

\section{Évolution saisonnière des paramètres}

La figure 1 indique la répartition sur les 13 mois de l'étude des maxima et minima obser- vés dans l'évolution des moyennes mensuelles des principaux paramètres de composition des laits de troupeau. Elle permet de distinguer 4 périodes dont l'alternance trace leurs cycles d'évolution annuelle: cycles longs et symétriques pour le TB et le lactose, avec un seul maximum annuel (automne pour le TB, printemps pour le lactose) ; cycles courts avec 2 maxima (fin d'été-début d'automne et printemps) pour le TP et les $\alpha-\mathrm{CN}, \beta-\mathrm{CN}, \kappa-\mathrm{CN}$. 
Les variations moyennes de chacune des $3 \mathrm{CN}$ mesurées et de leur somme peuvent être exprimées mathématiquement à partir des variations réelles, par le calcul des indices moyens mensuels de variation. La figure 2 montre le parallélisme d'évolution qui existe sur les 13 mois de l'étude entre les indices moyens de variation de la $\beta-C N$ et de la somme $\alpha-+\beta-+\kappa-C N$. Les corrélations $(n=13)$ calculées entre ces indices indiquent que c'est en effet la $\beta-\mathrm{CN}(r=0,930$, $P<0,001)$ qui apparaît comme le meilleur marqueur de l'évolution de la somme $\alpha-+\beta$ $+\kappa-\mathrm{CN}$, parmi les $3 \mathrm{CN}$ étudiées $(r=0,891$, $P<0,001$ et $r=0,599, P=0,05$ respectivement pour $\alpha-\mathrm{CN}$ et $\kappa-\mathrm{CN}$ ).

\section{Liens entre les paramètres}

Les liens de dépendance statistique entre les différents paramètres mesurés sur les laits de troupeau ont été estimés chaque mois et sur la durée totale de l'étude. La figure 3 indique que les concentrations des laits en $\alpha$ $\mathrm{CN}, \beta-\mathrm{CN}$ et $\kappa-\mathrm{CN}$ sont très fortement corrélées entre elles $(P<0,001)$. Les concentrations des laits en $\alpha-\mathrm{CN}, \beta-\mathrm{CN}$ et $\kappa$ $\mathrm{CN}$ sont également très fortement liées à la somme $\alpha-+\beta-+\kappa-C N(P<0,001)$. Mais la figure 4 qui montre l'évolution mensuelle des coefficients de corrélation calculés entre la somme $\alpha-+\beta-+\kappa-C N$ et les concentrations de la fraction $\alpha-\mathrm{CN}$ ou $\beta-\mathrm{CN}$ ou $\kappa-\mathrm{CN}$, indique que parmi ces $3 \mathrm{CN}$, c'est constamment l' $\alpha-C N$ qui est la mieux corrélée à leur somme et qui apparaît donc comme le meilleur marqueur de la concentration totale en $\mathrm{CN}$.

La somme $\alpha-+\beta-+\kappa-C N$ est encore corrélée $(P<0,001)$ sur la durée de l'étude aux TP, TB, lactose et à l'EST (fig 3). Cependant, la force de ces liens statistiques envisagés mois par mois, est moins constante que celle des liens observés entre les différentes CN. L'EST est très fortement corrélé aux TB et TP $(P<0,001)$. Lactose et EST,
TB et TP, lactose et TB sont corrélés 2 à 2 avec la même signification statistique mais des coefficients de corrélation un peu inférieurs. Enfin le lien observé entre lactose et TP est plus faible $(P=0,01)$.

\section{Classement relatif des troupeaux}

Un classement relatif des 50 troupeaux étudiés peut être élaboré en fonction de la valeur moyenne, déterminée sur la durée totale de l'étude, de la somme $\alpha-+\beta-+\kappa-\mathrm{CN}$ des laits produits. Il indique que seul un troupeau à race dominante FFPN figure parmi les 25 premiers classés. Treize de ces 25 meilleurs troupeaux sont à race Normande dominante. Les 11 autres sont des troupeaux mixtes, souvent à forte proportion de Normandes. Cependant, certains troupeaux, bien qu'exclusivement composés de vaches de race Normande, apparaissent mal positionnés.

Un écart très important est observé dans la valeur de la somme $\alpha-+\beta-+\kappa-C N$ entre les troupeaux extrêmes du classement $(24,7$ à $16,8 \mathrm{~g} / \mathrm{l})$. Une autre manière de considérer cet écart est de calculer, pour chaque troupeau, le volume moyen de lait contenant une quantité de $\mathrm{CN}$, exprimée en somme $\alpha-+\beta$ $+\kappa-\mathrm{CN}$, égale à $1 \mathrm{~kg}$. Pour l'ensemble des 50 troupeaux, ce volume est en moyenne de $51,1 \mathrm{I}$ avec un minimum égal à $40,4 \mathrm{I}$ et un maximum à $59,5 \mathrm{I}$. Un écart de $19,1 \mathrm{I} \mathrm{par} \mathrm{kg}$ de $\mathrm{CN}$ sépare ainsi les 2 troupeaux extrêmes du classement et correspond, dans ce cas précis, à un volume supplémentaire de plus de 60000 I de lait livré en une année à la laiterie pour une même quantité de $\mathrm{CN}$ $(3500 \mathrm{~kg})$. Pour l'ensemble des troupeaux étudiés, les 25 premiers classés ont livré sur les 13 mois de l'étude, 96 tonnes de $\mathrm{CN}$ pour $4,710^{6}$ I de lait; les 25 autres, 98 tonnes de CN dans $5,310^{6} 1$ de lait, soit seulement 2 tonnes supplémentaires de $\mathrm{CN}$ pour une différence de 600000 I de lait collectés. 
Tableau II. Qualité potentielle des herbages en fonction de la nature et des caractéristiques des sols rencontrés dans la région d'élevage des troupeaux étudiés.

Potential quality of grass-lands according to the nature and characteristics of the soils inventoriated in the breeding area of studied herds.

\begin{tabular}{|c|c|}
\hline Sol & Caractéristiques et qualité de l'herbage \\
\hline Dunaires & $\begin{array}{l}\text { Affleurement de sable (dunes quaternaires), sec et sans remontée capillaire } \\
\text { d'eau, peu favorable à un herbage de qualité }\end{array}$ \\
\hline Rendzine brune & $\begin{array}{l}\text { Mull riche en calcium, sur Hettangien et Sinémurien marno-calcaire, favorable } \\
\text { à un herbage de qualité }\end{array}$ \\
\hline Brun calcaire & $\begin{array}{l}\text { Mull riche en calcaire actif, suffisamment humide, sur Hettangien et Sinémurien } \\
\text { marno-calcaire, très favorable à un herbage de bonne qualité }\end{array}$ \\
\hline Brun eutrophe & $\begin{array}{l}\text { Mull peu humifère neutre, d'humidité moyenne, sur Hettangien et Sinémurien } \\
\text { marno-calcaire, très favorable à un herbage de bonne qualité }\end{array}$ \\
\hline Brun lessivé & $\begin{array}{l}\text { Mull mésotrophe d'humidité moyenne, sur poudingues, schistes et grès } \\
\text { primaires, encore favorable à un herbage de qualité } \\
\text { Mull mésotrophe moins riche en calcium que le précédent, sur sables coquilliers } \\
\text { et faluns du Miocène-Pliocène, peu favorable à un herbage de qualité }\end{array}$ \\
\hline Lessivé & $\begin{array}{l}\text { Mull-moder à Moder, plus acide et moins décomposé que le précédent, peu } \\
\text { favorable à un herbage de qualité. }\end{array}$ \\
\hline Podzoliques & $\begin{array}{l}\text { Mor acide à matière organique mal ou pas décomposée, peu favorable voire } \\
\text { défavorable à un herbage de qualité }\end{array}$ \\
\hline Alluviaux & $\begin{array}{l}\text { Sur alluvions quaternaires, favorables à un herbage de très bonne qualité si la } \\
\text { nappe phréatique oscille suffisamment (drainage naturel ou artificiel) } \\
\text { Peu favorables si la nappe oscille peu (bas fonds) ou en cas d'Hydromull sur } \\
\text { alluvions tourbeuses (transgression marine récente) }\end{array}$ \\
\hline
\end{tabular}

Facteurs qui déterminent à la production les paramètres de composition des laits de troupeau

\section{Race}

La race Normande apparaît significativement favorable (tableau III et fig 5 ) avec des écarts moyens sur les 13 mois de l'étude de $1,6 \mathrm{~g} / \mathrm{l}$ pour la somme $\alpha-+\beta-+\kappa-\mathrm{CN}(P<$ $0,001)$, le TP $(P<0,001)$ et le TB $(P=0,05)$ et de $3,3 \mathrm{~g} / \mathrm{l}$ pour l'EST $(P<0,001)$. Le rapport somme $\alpha-+\beta-+\kappa-C N / T P$ dans le lait des troupeaux à race Normande dominante est également supérieur (2\%) à celui obtenu pour les troupeaux à race FFPN dominante. La prééminence de la race Normande sur la race FFPN doit cependant être pondérée se-
Ion les mois et les paramètres. Pour tous les paramètres, les écarts observés varient en effet avec les saisons et ils apparaissent globalement plus faibles en automne-hiver qu'au printemps-été. Ainsi, atteignant par exemple 2,7 g/l en juin 1993, l'écart pour la somme $\alpha-+\beta-+\kappa-C N$ est peu supérieur à $1 \mathrm{~g} / \mathrm{l}$ en novembre et décembre 1992 , tandis que pour le TB, il passe de $0,4 \mathrm{~g} / \mathrm{l}$ en novembre 1992 (NS à $P=0,1$ ) à $2,5 \mathrm{~g} / \mathrm{l}$ en avril et juillet 1993. Le lactose est le moins influencé des paramètres étudiés et, certains mois, sa concentration est même supérieure dans le lait produit par les FFPN. Les valeurs mesurées pour les mêmes paramètres dans les troupeaux mixtes sont intermédiaires (tableau I) entre celles observées dans les troupeaux à race dominante Normande et FFPN. 


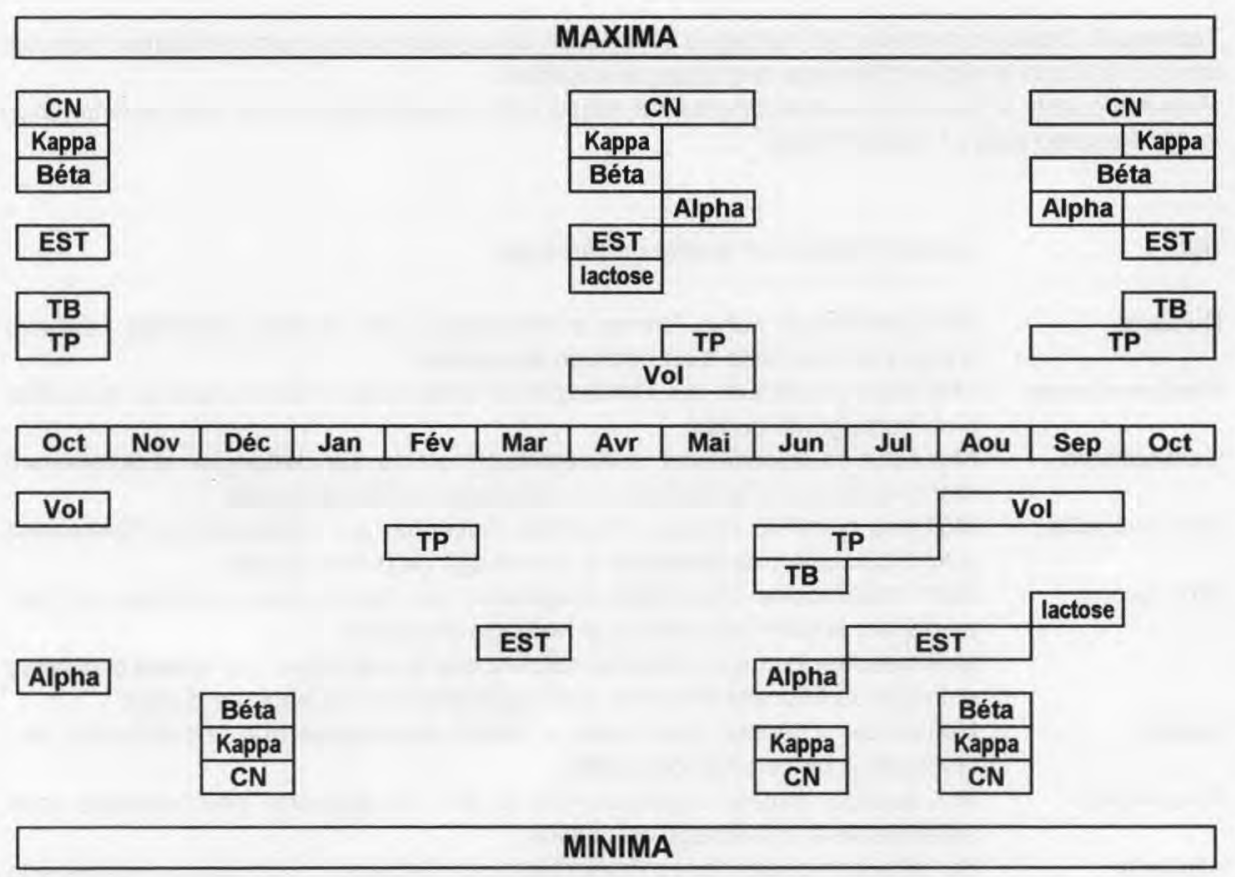

Fig 1. Répartition saisonnière des maxima et minima observés dans l'évolution des moyennes mensuelles des paramètres mesurés sur les laits de troupeau. (Vol : volume de lait produit par $24 \mathrm{~h}$, $\mathrm{CN}$ : somme $\alpha-+\beta-+\kappa-C N$, TP : taux protéique, TB : taux butyreux, EST : extrait sec total).

Seasonal distribution of the maxima and minima observed for the monthly averages of parameters measured in herd milks. (Vol: volume of milk per $24 \mathrm{~h}, \mathrm{CN}$ : total amount $\alpha-+\beta-+\kappa-C N$, TP: proteins, TB: fat, EST: dry matter).

\section{Sol}

Chacun des sols inventoriés dans la région d'élevage des troupeaux étudiés, possède une potentialité à produire un herbage dont la qualité, en relation avec sa composition floristique, est liée à sa nature et à ses caractéristiques (tableau II). Pour l'étude de l'influence du sol sur les paramètres mesurés sur les laits, les troupeaux concernés par des sols très favorables ( 11 troupeaux sur sols brun calcaire et brun eutrophe) et des sols favorables à un herbage de qualité (18 troupeaux sur rendzine et sol brun lessivé sur primaire) sont regroupés et comparés aux troupeaux localisés sur sols peu favorables (12 troupeaux sur dunes, sol brun lessivé sur tertiaire, sol lessivé ou sols podzoliques). Les valeurs moyennes des paramètres de composition mesurés sur les laits de ces groupes sont indiquées dans le tableau III. Des différences significatives pour EST $(P<0,001)$, TB $(P=0,05)$ et la somme $\alpha-+\beta-+\kappa-\mathrm{CN}(P$ $=0,01$ ) sont observées entre le groupe de troupeaux sur sols peu favorables et celui sur sols très favorables ou favorables à un herbage de qualité (fig 6 ). Cet effet du sol n'est que peu significatif pour le lactose $(P=0,1)$ et ne l'est pas pour le TP. Les sols alluviaux, dont les potentialités liées à l'aération suffi- 
Tableau III. Influence de la potentialité pédologique des sols, de la race dominante des troupeaux, de la répartition saisonnière des vêlages, de l'importance de l'ensilage de maïs dans l'alimentation en stabulation et de la constance de l'alimentation complémentaire sur les paramètres de composition des laits.

Effect of the pedologic potentialities of soils, dominant breed of herds, seasonal distribution of calving, com ensilage feed during stabbling and maintenance of complementary feeding, on the compounds of herd milks.

\begin{tabular}{|c|c|c|c|c|c|c|c|c|c|c|c|}
\hline \multirow[t]{2}{*}{ Facteur } & \multirow[t]{2}{*}{$n^{1}$} & \multicolumn{2}{|c|}{$\operatorname{TP}(g / l)$} & \multicolumn{2}{|c|}{$T B(g / l)$} & \multicolumn{2}{|c|}{ Lactose $(g / l)$} & \multicolumn{2}{|c|}{$E S T(g / l)$} & \multicolumn{2}{|c|}{$C N^{2}(g / l)$} \\
\hline & & Moy & $E T^{3}$ & Moy & $E T$ & Moy & $E T$ & Moy & $E T$ & Moy & $E T$ \\
\hline \multicolumn{12}{|l|}{ Pédologie } \\
\hline Favorable & 29 & 33,1 & 1,0 & 43,4 & 2,3 & 49,4 & 0,6 & 133,3 & 3,3 & 20,0 & 1,3 \\
\hline Peu favorable & 12 & 32,6 & 1,1 & 41,9 & 2,0 & 48,9 & 0,5 & 130,7 & 3,0 & 18,8 & 1,2 \\
\hline \multicolumn{12}{|l|}{ Race } \\
\hline Normande & 19 & 33,5 & 1,2 & 43,3 & 3,4 & 49,3 & 0,7 & 133,7 & 4,6 & 20,3 & 1,6 \\
\hline FFPN & 12 & 31,9 & 0,5 & 41,7 & 1,5 & 49,0 & 0,5 & 130,4 & 2,1 & 18,7 & 0,5 \\
\hline \multicolumn{12}{|l|}{ Vêlages } \\
\hline \multicolumn{12}{|l|}{ Automne- } \\
\hline hiver & 27 & 33,0 & 1,2 & 43,6 & 2,6 & 49,3 & 0,7 & 133,4 & 4,0 & 20,0 & 1,4 \\
\hline Autre & 22 & 32,6 & 1,1 & 42,0 & 1,9 & 48,9 & 0,6 & 131,2 & 2,7 & 19,0 & 1,1 \\
\hline \multicolumn{12}{|l|}{$\begin{array}{l}\text { Ensilage } \\
\text { maïs }\end{array}$} \\
\hline Principal & 35 & 32,9 & 1,2 & 43,4 & 2,5 & 49,3 & 0,7 & 133,0 & 3,8 & 19,8 & 1,4 \\
\hline Secondaire & 14 & 32,8 & 1,1 & 41,5 & 1,7 & 48,7 & 0,4 & 130,9 & 2,3 & 19,0 & 1,2 \\
\hline \multicolumn{12}{|l|}{ Complément } \\
\hline Constant & 29 & 33,1 & 1,3 & 43,5 & 2,7 & 49,4 & 0,7 & 133,5 & 4,1 & 19,9 & 1,6 \\
\hline Inconstant & 20 & 32,5 & 0,7 & 41,9 & 1,5 & 48,8 & 0,5 & 130,8 & 2,0 & 19,1 & 0,7 \\
\hline
\end{tabular}

${ }^{1} \mathrm{n}$ : nombre de troupeaux $;{ }^{2} \mathrm{CN}$ : somme $\alpha-+\beta-+\kappa-\mathrm{CN} ;{ }^{3} \mathrm{ET}$ : écart type.

sante de l'humus sont conditionnées par l'efficacité du drainage, naturel ou artificiel, à rabattre suffisamment la nappe phréatique, ne sont pas considérés ( 9 troupeaux).

\section{Répartition saisonnière des vêlages}

La répartition des vêlages dans l'année constitue un facteur lié à la conduite du troupeau déterminant des paramètres de composition du lait. Pour 27 troupeaux sur 49
(55\%), les vêlages sont principalement situés en automne et en hiver. Pour les 22 autres, ils sont généralement répartis de façon homogène sur l'année. Les résultats (tableau III et fig 6) montrent l'influence favorable des vêlages d'automne-hiver sur le TB et l'EST $(P<0,001)$, la somme $\alpha-+\beta-+\kappa-$ CN $(P=0,01)$ et le lactose $(P=0,1)$ du lait produit. Comme pour d'autres facteurs, l'influence sur le TP n'apparaît, en revanche, pas significative. 
Tableau IV. Valeurs minimales, maximales et moyennes des paramètres mesurés sur les laits de fromagerie crus et standardisés.

General statistics of parameters assayed in raw and fat-standardized dairy-milks.

\begin{tabular}{|c|c|c|c|c|c|c|c|c|}
\hline & \multicolumn{4}{|c|}{ Laits crus } & \multicolumn{4}{|c|}{ Laits standardisés } \\
\hline & Min & Max & Moy & $E T^{5}$ & Min & $\operatorname{Max}$ & Moy & ET \\
\hline $\operatorname{Tf}^{1}(\min )$ & 4,2 & 6,1 & 5,1 & 0,5 & 4,9 & 6,7 & 5,7 & 0,5 \\
\hline $\mathrm{K} 15^{2}$ (min) & 5,6 & 11,7 & 8,0 & 2,0 & 7,0 & 17,8 & 10,6 & 3,2 \\
\hline $\mathrm{A} 30^{3}(\mathrm{~mm})$ & 18,3 & 23,3 & 20,9 & 1,7 & 15,6 & 22,6 & 19,8 & 2,3 \\
\hline$\alpha-C N(g / l)$ & 8,8 & 11,0 & 9,8 & 0,6 & 9,8 & 11,6 & 10,3 & 0,5 \\
\hline$\beta-C N(g / l)$ & 5,9 & 7,2 & 6,3 & 0,3 & 6,0 & 7,3 & 6,6 & 0,4 \\
\hline$\kappa-C N(a / l)$ & 2,2 & 2,9 & 2,5 & 0,2 & 2,5 & 3,2 & 2,7 & 0,2 \\
\hline $\mathrm{CN}^{4}(\mathrm{~g} / \mathrm{l})$ & 17,2 & 20,1 & 18,6 & 0,9 & 18,5 & 21,1 & 19,7 & 0,7 \\
\hline $\operatorname{TP}(g / l)$ & 31,3 & 33,3 & 32,4 & 0,6 & 31,2 & 33,2 & 32,3 & 0,6 \\
\hline TB $(g / l)$ & 29,8 & 43,1 & 38,5 & 4,4 & 23,1 & 24,1 & 23,6 & 0,3 \\
\hline EST $(g / l)$ & 119,4 & 131,7 & 126,8 & 4,1 & 109,7 & 115,8 & 113,3 & 1,9 \\
\hline Ca total $(g / l)$ & 1,22 & 1,34 & 1,27 & 0,04 & 1,2 & 1,3 & 1,29 & 0,03 \\
\hline
\end{tabular}

${ }^{1}$ Tf : temps de floculation : ${ }^{2} \mathrm{~K} 15$ : indice de raffermissement $;{ }^{3} \mathrm{~A} 30$ : indice de fermeté $;{ }^{4} \mathrm{CN}$ : somme $\alpha-+\beta-+\kappa-C N ;{ }^{5}$ ET : écart type.

\section{Aspect quantitatif de l'alimentation}

Des corrélations positives, faibles mais significatives $(P=0,05)$, sont obtenues entre la ration alimentaire journalière et le TB $(r=0,319)$, le lactose $(r=0,302)$, la somme $\alpha-+\beta-+\kappa-C N(r=0,287)$ et l'EST $(r=0,279)$ mesurés dans les laits des 50 troupeauxétudiés. Aucun lien significatif n'est par contre retrouvé avec le TP. Une alimentation moyenne significativement moindre ($0,9 \mathrm{~kg} / \mathrm{vache} / \mathrm{jour}, P=0,05)$ des troupeaux à race Normande dominante par rapport aux troupeaux à race FFPN dominante est par ailleurs observée. Les vaches Normandes semblent donc produire un lait plus riche que les FFPN, tout en étant quantitativement moins alimentées. Le rapport somme $\alpha-+$ $\beta-+\kappa-C N /$ ration alimentaire, qui représente en quelque sorte un indice de production de la vache, est de ce fait supérieur $(P<0,001)$ chez les 19 troupeaux à race
Normande dominante $(1,27$, écart type $(E T)=0,08$ contre $1,11, E T=0,07$ dans les 13 troupeaux à race FFPN dominante).

\section{Aspect qualitatif de l'alimentation}

L'analyse des données qualitatives de l'alimentation des troupeaux montre l'importance sur les paramètres de composition du lait produit, de 2 facteurs principaux : l'ensilage de maïs en stabulation et l'alimentation complémentaire.

L'ensilage de maïs constitue l'aliment principal en stabulation pour $35(71 \%)$ troupeaux, dont 11 troupeaux sur les 12 à race FFPN dominante et 12 sur les 18 à race Normande dominante. Pour les 14 autres, il est absent de cette alimentation ou simplement un aliment secondaire aux côtés le plus souvent du foin ou de l'ensilage d'herbe. Le tableau III et la figure 6 indiquent le caractère favorable de cette présence de l'ensilage de 


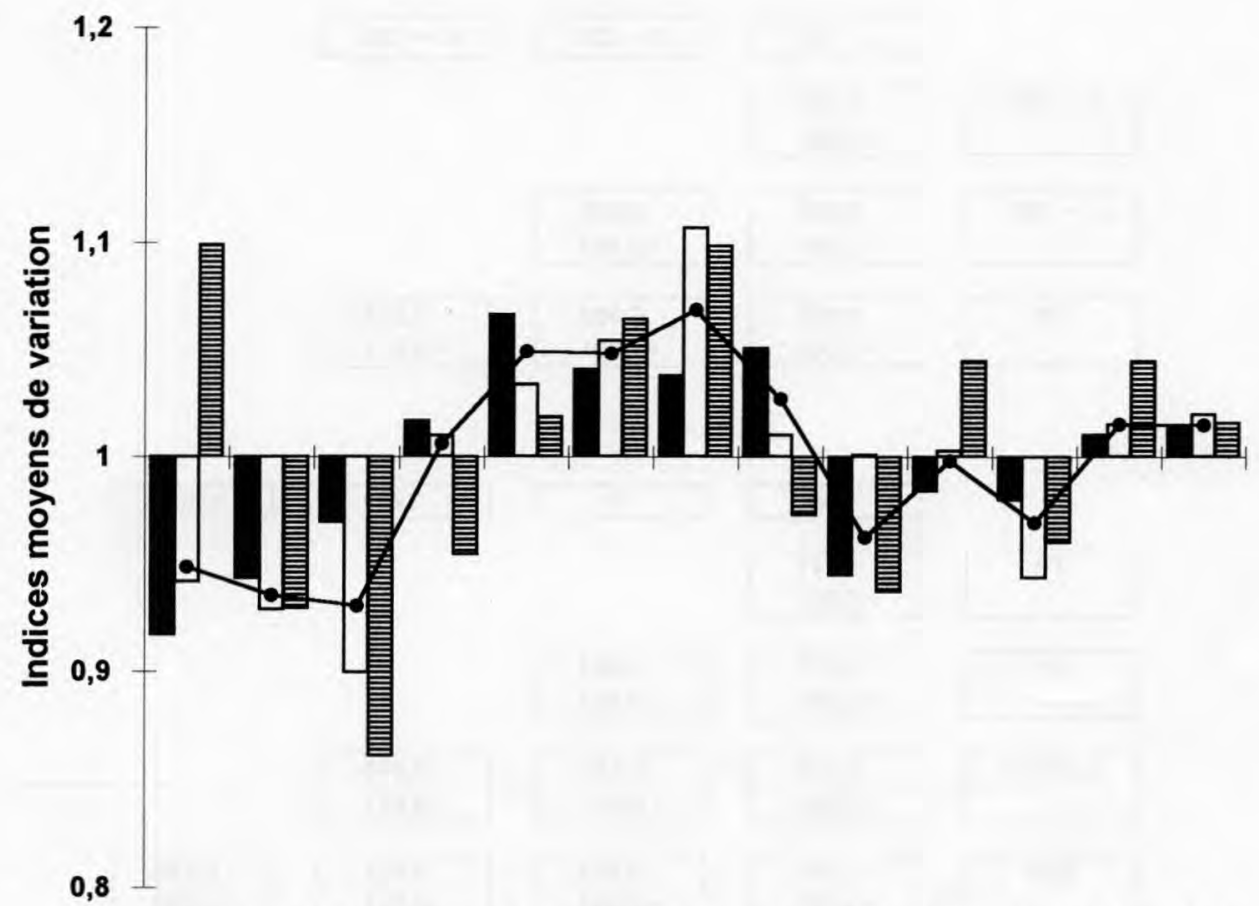

\section{Oct Nov Déc Jan Fév Mar Avr Mai Jun Jul Aou Sep Oct Mois}

Fig 2. Évolution mensuelle comparée des indices moyens de variation calculés pour les $\alpha$-CN (घ), $\beta-\mathrm{CN}(\square), \kappa-\mathrm{CN}$ (圆) et la somme $\alpha-+\beta-+\kappa-\mathrm{CN}(\bullet)$.

$\alpha-\mathrm{CN}(\boldsymbol{\bullet}), \beta-\mathrm{CN}(\square), \kappa-\mathrm{CN}($ 国) and the total amount $\alpha-+\beta-+\kappa-\mathrm{CN}(\bullet)$.

maïs comme aliment principal en stabulation sur le TB et l'EST $(P<0,001)$, la somme $\alpha$ $+\beta-+\kappa-\mathrm{CN}$ et le lactose $(P=0,05)$. L'influence sur le TP n'est en revanche pas significative.

L'alimentation complémentaire est quelquefois à base d'aliments auto-produits (foin, blé, orge, avoine) mais le plus souvent, elle est essentiellement assurée par des aliments concentrés dont la nature et la valeur nutritionnelle sont inconnues.

L'alimentation complémentaire est constante sur les 13 mois de l'étude pour 29 troupeaux $(59 \%)$, inconstante pour les autres. Les résultats du tableau III et de la fig. 6 montrent le caractère favorable de cette constance de l'alimentation complémentaire pour le TB et l'EST $(P<0,001)$, la somme $\alpha$ $+\beta-+\kappa-\mathrm{CN}$ et le lactose $(P=0,05)$, le TP $(P=$ $0,1)$.

\section{Transitions entre stabulation et pâturage}

L'influence de la durée de la transition stabulation - pâturage et les effets de celle-ci sur la somme $\alpha-+\beta-+\kappa-\mathrm{CN}$ du lait varient de façon significative $(P<0,001)$ selon la nature de l'aliment principal donné au troupeau en stabulation (fig 6). Quand l'ensilage de maïs est 


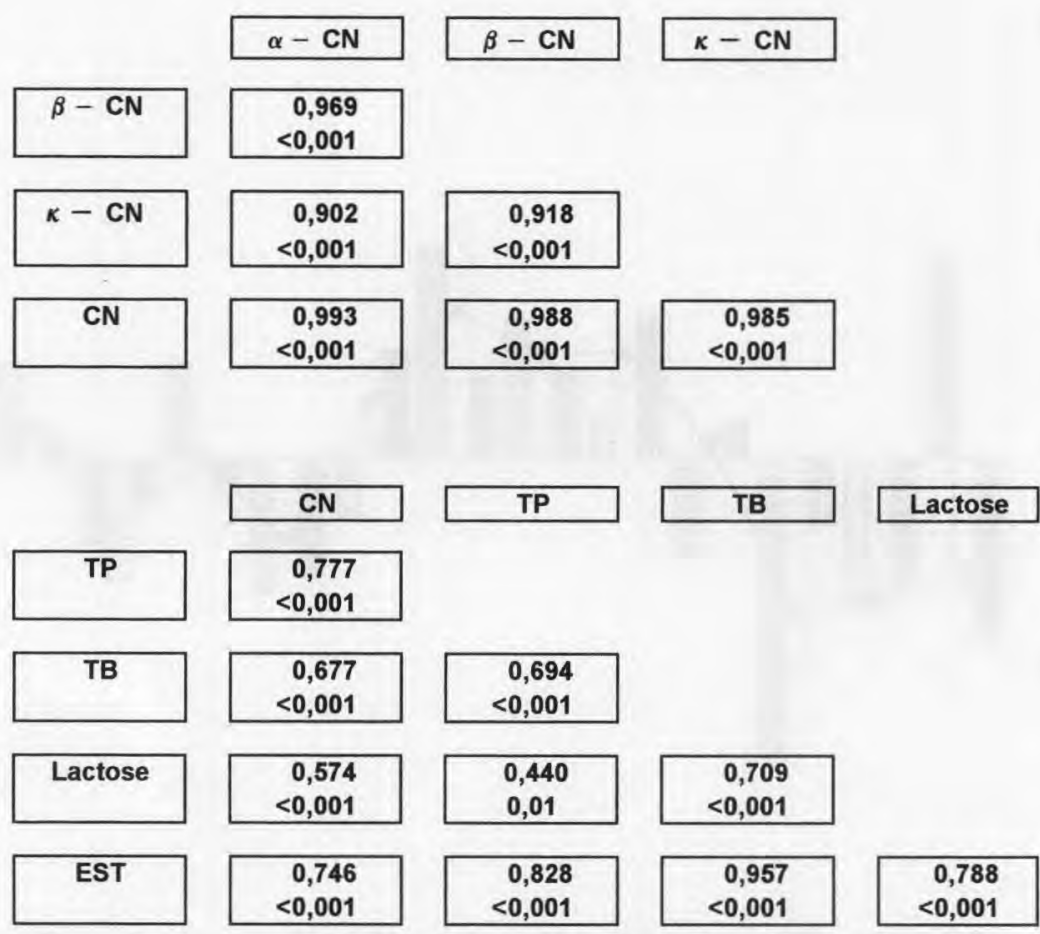

Fig 3. Corrélations (coefficients de corrélation et signification) entre les paramètres mesurés sur les laits de troupeau. ( $\mathrm{CN}$ : somme $\alpha-+\beta-+\kappa-\mathrm{CN})$.

Correlations (correlation coefficients and signification) between the parameters measured in herd milks. (CN: total amount $\alpha-+\beta-+\kappa-C N)$.

absent ou secondaire dans l'alimentation en stabulation, c'est-à-dire quand celle-ci est basée principalement sur le foin ou l'ensilage d'herbe (14 troupeaux), le passage au pâturage se traduit par une augmentation $(3,1 \%$, $E T=0,1)$ de la somme $\alpha-+\beta-+\kappa-C N$ non expliquée par les variations moyennes. En revanche, quand l'ensilage de maïs constitue l'aliment principal (35 troupeaux), une diminution est globalement notée $(-0,7 \%$, $E T=1,9)$. Dans ce dernier cas, quand la durée de la transition est inférieure à 6 semaines (12 troupeaux) la diminution de la somme $\alpha-+\beta-+\kappa-C N$ après correction des variations moyennes est forte $(-2,0 \%, E T=0,1)$. Elle est nettement plus faible $(-0,1 \%$,
$E T=0,1)$ pour une transition longue, égale ou supérieure à 6 semaines ( 23 troupeaux).

A l'inverse de la transition stabulation-pâturage, aucune tendance significative n'a pu être établie pour la transition pâturage-stabulation. Lors de celle-ci, la diminution de la quantité et de la qualité de l'herbe disponible est généralement compensée par un apport de foin, d'ensilage d'herbe ou d'ensilage de maïs, prémices de l'alimentation en stabulation.

\section{Étude individuelle des troupeaux}

Seuls les résultats de l'étude globale des facteurs qui déterminent les paramètres de composition du lait à la production ont été 


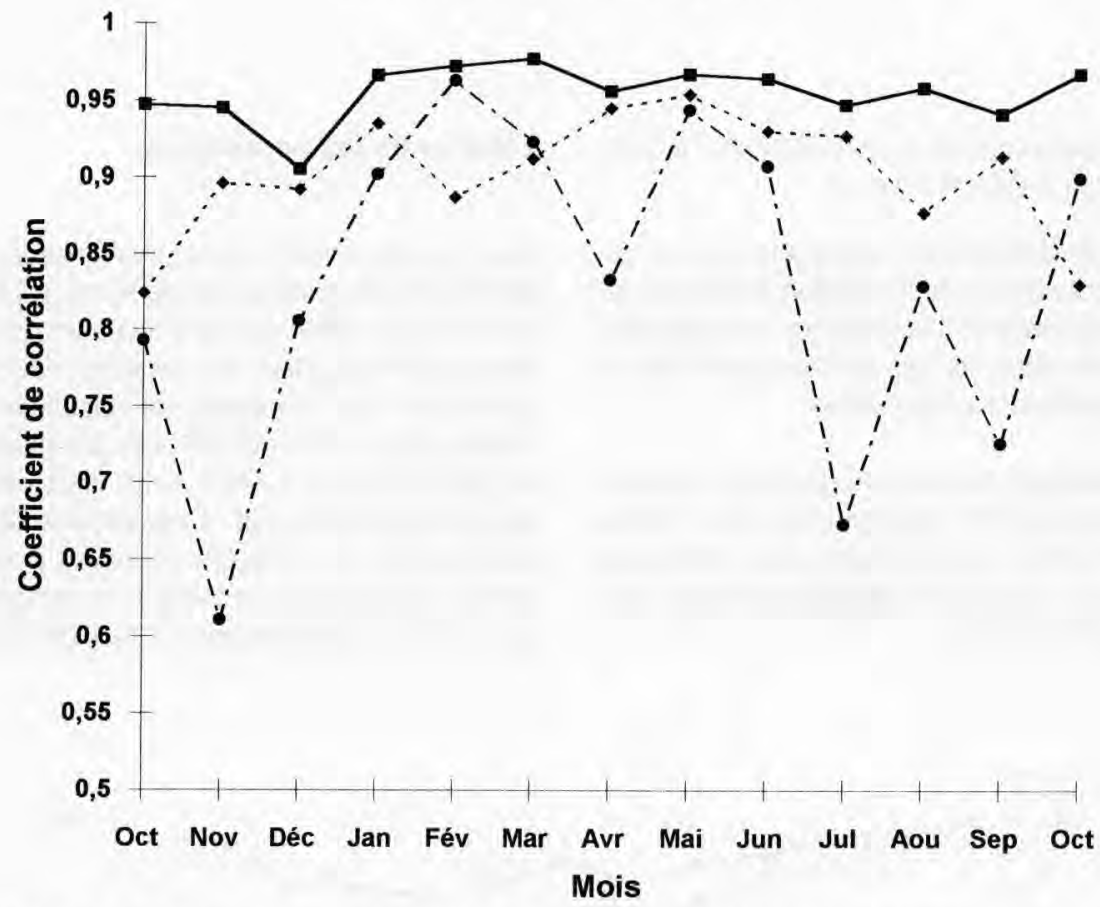

Fig 4. Évolution mensuelle des coefficients de corrélation calculés entre la somme $\alpha-+\beta-+\kappa-\mathrm{CN}$ et $\alpha-\mathrm{CN}(\boldsymbol{\bullet}), \beta-(\bullet), \kappa-\mathrm{CN}(\bullet)$.

Monthly changes of the correlation coefficients calculated between the total amount $\alpha-+\beta-+\kappa-C N$ and $\alpha-C N(\bullet), \beta-(\bullet), \kappa-C N(\bullet)$.

pris jusqu'à présent en considération. Comme indiqué dans Matériels et méthodes, ces facteurs ont été définis lors d'une étude individuelle préalable des conditions de production, effectuée troupeau par troupeau. II est maintenant possible de revenir à cette étude individuelle, en exprimant, pour chacun des 50 troupeaux, l'état favorable ou non de ces facteurs.

La figure 7 propose une synthèse de ces analyses individuelles en fonction du classement général annuel des troupeaux, selon la somme $\alpha-+\beta-+\kappa-\mathrm{CN}$ mesurée dans leur lait.

Elle montre la fréquence du cumul des facteurs favorables pour les troupeaux les mieux classés, puis un gradient, fonction du classement, de l'état de ces facteurs, d'abord favorable puis de plus en plus souvent défavorable et qui aboutit à une plus grande fré- quence des facteurs défavorables pour les troupeaux les plus mal positionnés.

\section{Étude des laits destinés à la fromagerie}

\section{Étude globale des paramètres}

Les valeurs obtenues pour les paramètres de composition des laits de fromagerie crus (tableau IV) sont plus faibles (2 à 10\%) que celles des laits de troupeau précédemment étudiés (tableau I), avec des différences significatives pour l'a-CN, la somme $\alpha-+\beta-+$ $\kappa-C N$, le TB et l'EST $(P<0,001)$, le TP ( $P$ $=0,05)$, et la $\beta$-CN $(P=0,1)$. La diminution du TB et de l'EST (respectivement 39 et $11 \%, P<0,001$ ) et la réduction de la dispersion des TB dans les laits standardisés s'accompagnent de l'augmentation (5 à $8 \%$ ) de l' 
$\alpha-\mathrm{CN}$, de la somme $\alpha-+\beta-+\kappa-\mathrm{CN}(P<0,001)$, $\operatorname{des} \beta$ - et $\kappa-\mathrm{CN}(P=0,01)$.

Les évolutions des paramètres de la coagulation, temps de floculation, indice de raffermissement $\mathrm{K} 15$ et indice de fermeté $\mathrm{A} 30$, mesurés dans les laits de fromagerie crus et standardisés sont parallèles.

Cependant, les valeurs du temps de floculation et du $\mathrm{K} 15$ sont toujours plus faibles $(P<0,001)$ dans les laits crus, tandis que celles du $\mathrm{A} 30$ y sont généralement plus élevées $(P<0,001)$.

\section{Liens entre les paramètres}

Que les laits soient crus ou standardisés en MG (fig 8), les paramètres de la coagulation sont corrélés entre eux, positivement pour le temps de floculation et le coefficient $K 15$, négativement pour le temps de floculation et l'indice A30, le K15 et l'A30. Les corrélations significatives qui existent entre paramètres de composition du lait et paramètres de sa coagulation sont toujours négatives pour le temps de floculation et l'indice de raffermissement K15, positives pour l'indice de ferme-

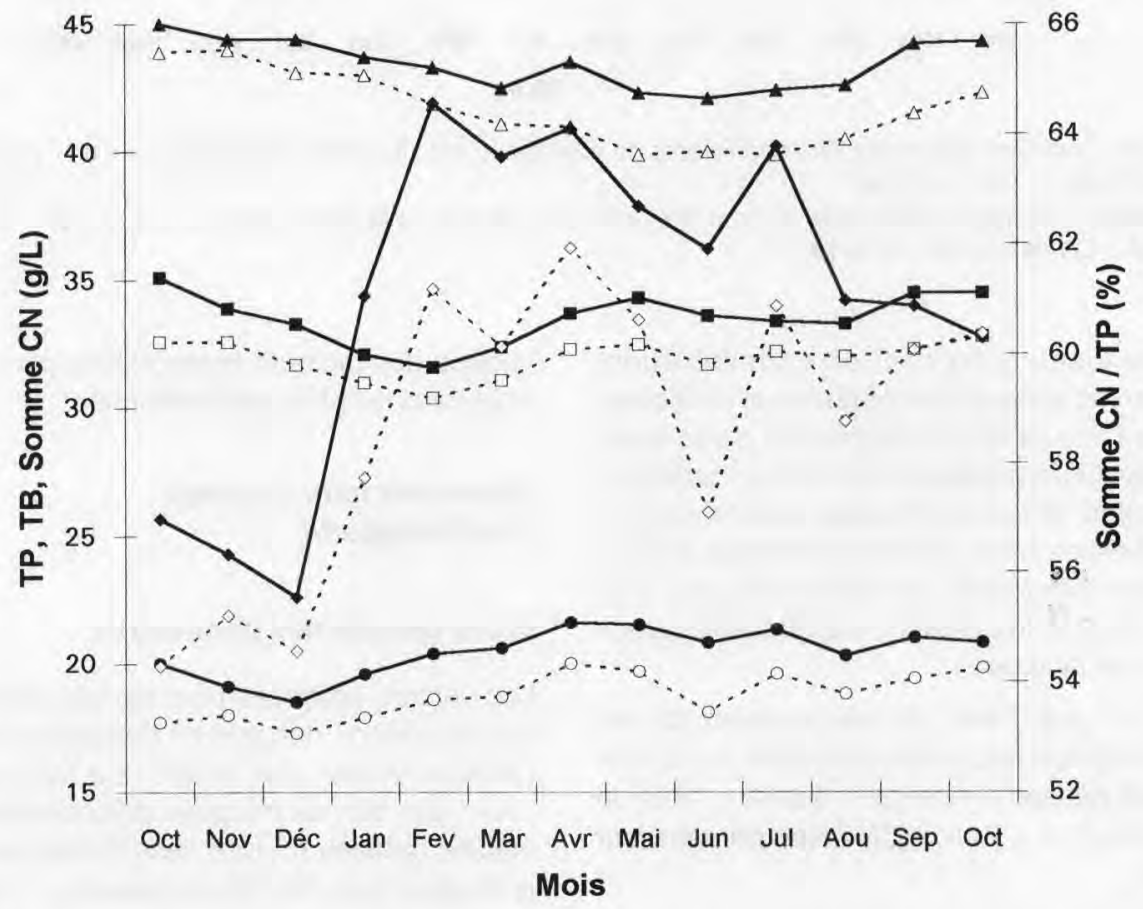

Fig 5. Évolution mensuelle comparée des $\operatorname{TP}(\boldsymbol{\bullet}, \square)$, TB $(\boldsymbol{\Delta}, \Delta)$, de la somme $\alpha-+\beta-+\kappa-\mathrm{CN}(\bullet, 0)$ et du rapport somme $\alpha-+\beta-+\kappa-\operatorname{CN} / \operatorname{TP}(\bullet, 0)$ dans le lait des troupeau à race Normande dominante $(\bullet, \Delta, \bullet)$ et à race FFPN dominante $(\square, \Delta, O, 0)$.

Comparison of the monthly changes of TP $(\square, \square), T B(\boldsymbol{\Delta}, \Delta)$, total amount $\alpha-+\beta-+\kappa-C N(\bullet, 0)$ and ratio total amount $\alpha-+\beta-+\kappa-C N / T P(\bullet, 0)$ in the milks of Normande dominant-breed $(\mathbf{m , \Delta ,} \bullet \bullet)$ and FFPN dominant-breed herds $(\square, \Delta, 0, \diamond)$. 


\section{EXPLOITATION}

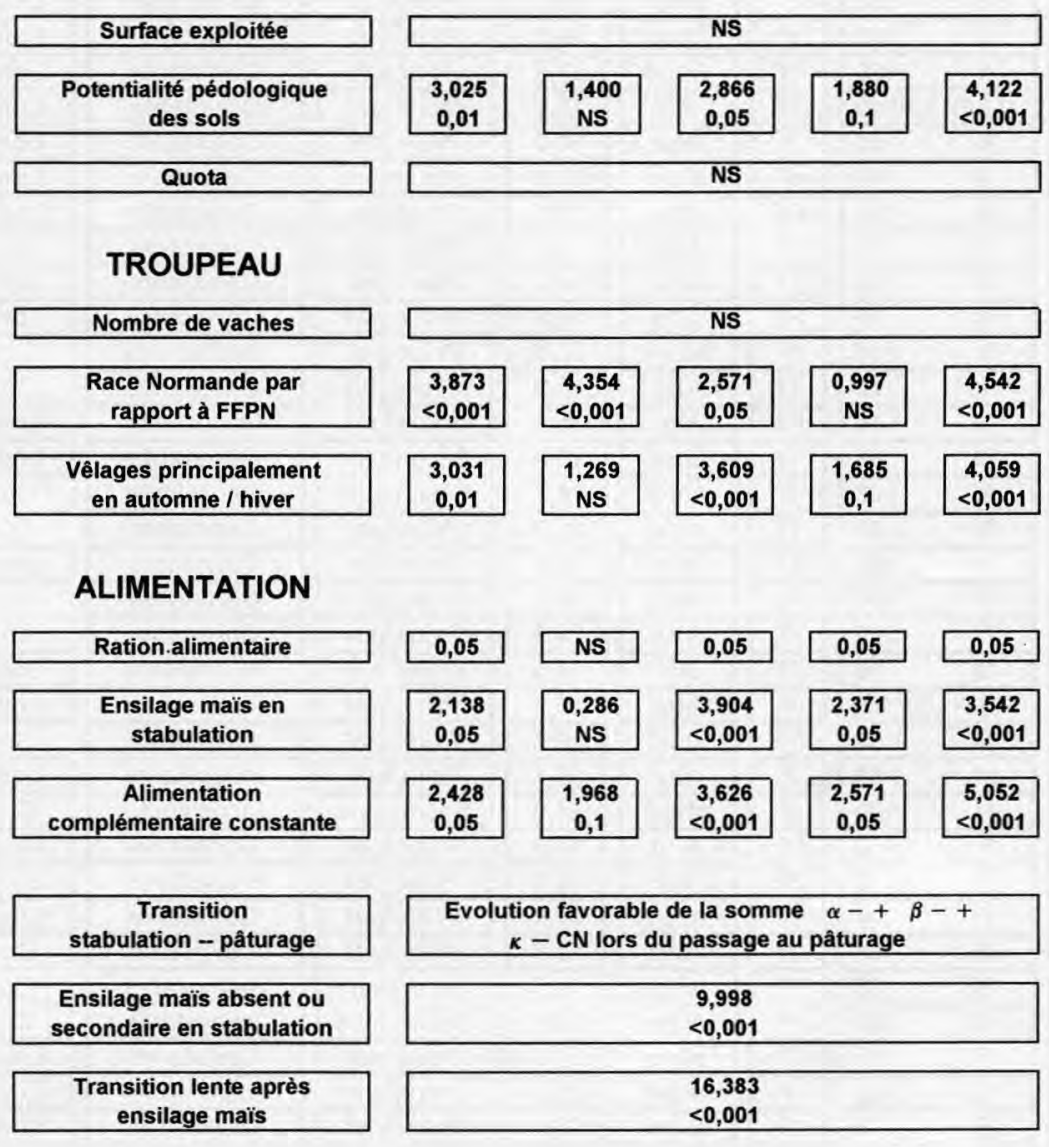

Fig 6. Influence relative (écart t de Student et signification) des facteurs qui déterminent les paramètres de qualité du lait à la production. (CN : somme $\alpha-+\beta-+\kappa-C N$; NS : non significatif à $P<0,1)$. Relative influence (coefficient of the Student's t-test and signification) of the factors defining quality parameters in herd milks. (CN: total amount $\alpha-+\beta-+\kappa-C N$; NS: not significant at $\mathrm{P}<0.1$ ).

té $\mathrm{A} 30$. Les paramètres de la coagulation mesurés dans les laits standardisés en MG apparaissent fortement liés à la $\beta-\mathrm{CN}$ et à la somme $\alpha-+\beta-+\kappa-C N(P<0,001)$, au TP et, malgré la standardisation, au TB $(P<0,001$ avec K15 et A30). Les corrélations obtenues dans les laits crus sont plus faibles que celles observées dans les laits standardisés en 


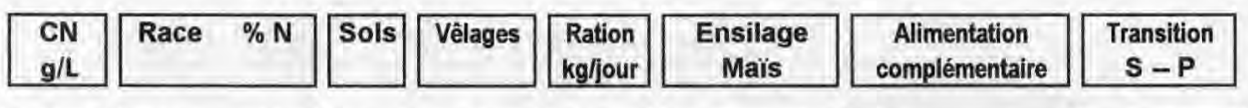

\begin{tabular}{|c|c|c|}
\hline 24,7 & 100 & $\mathrm{~F}$ \\
\hline 22,1 & 100 & $F$ \\
\hline 21,9 & 100 & TF \\
\hline 21,6 & $\mathrm{~N} / \mathrm{M} / 100 / 50$ & TF \\
\hline 21,3 & $\begin{array}{ll}N & 100 \\
\end{array}$ & $F$ \\
\hline 21,2 & 100 & $F$ \\
\hline 21,2 & 100 & $\mathrm{PF}$ \\
\hline 20,6 & 75 & FS \\
\hline 20,5 & 100 & TF \\
\hline 20,4 & 75 & $\mathrm{PF}$ \\
\hline 20,2 & 100 & $F$ \\
\hline 20,2 & 90 & TF \\
\hline 20,1 & 75 & $F S$ \\
\hline 20,1 & 66 & TF \\
\hline 20,1 & 100 & $F$ \\
\hline 20,0 & 66 & TF \\
\hline 20,0 & 66 & TF \\
\hline 19,8 & 100 & FS \\
\hline 19,7 & 100 & $\mathrm{~F}$ \\
\hline 19,7 & M $\quad 50$ & TF \\
\hline 19,7 & $M / N 50 / 100$ & $F$ \\
\hline 19,6 & $\begin{array}{ll}M & 50 \\
\end{array}$ & $\mathrm{FS}$ \\
\hline 19,5 & 66 & $F$ \\
\hline 19,5 & 100 & $F$ \\
\hline 19,4 & 0 & $\mathrm{PF}$ \\
\hline 19,3 & $\mathrm{~F}$ & FS \\
\hline 19,3 & $\mathrm{~F}$ & $\mathrm{PF}$ \\
\hline 19,2 & 66 & $F$ \\
\hline 19,2 & 80 & $\mathrm{PF}$ \\
\hline 19,2 & 100 & $\mathrm{PF}$ \\
\hline 19,1 & 15 & $F$ \\
\hline 19,0 & 100 & $F$ \\
\hline 19,0 & 0 & $F$ \\
\hline 18,9 & 40 & $\mathrm{~F}$ \\
\hline 18,9 & 85 & $\mathrm{PF}$ \\
\hline 18,8 & 15 & TF \\
\hline 18,8 & 100 & $F$ \\
\hline 18,7 & 50 & $F$ \\
\hline 18,6 & $\mathrm{~F}$ & $\mathrm{FS}$ \\
\hline 18,6 & 10 & TF \\
\hline 18,5 & $\mathrm{~F}$ & $\mathrm{~F}$ \\
\hline 18,5 & 40 & FS \\
\hline 18,4 & 80 & $\mathrm{TF}$ \\
\hline 18,4 & $\mathrm{~F}$ & $\mathrm{FS}$ \\
\hline 18,3 & $\mathrm{~F}$ & $\mathrm{PF}$ \\
\hline 18,0 & 10 & $\mathrm{PF}$ \\
\hline 18,0 & 100 & FS \\
\hline 17,7 & 0 & $\mathrm{PF}$ \\
\hline 17,3 & 50 & PF \\
\hline 16,8 & 33 & $\mathrm{PF}$ \\
\hline
\end{tabular}

\begin{tabular}{|c|c|}
\hline $\mathrm{aHp}$ & 18,5 \\
\hline Ahpé & 16,3 \\
\hline Ahpé & 17,0 \\
\hline Ah & 16,8 \\
\hline Ahp & 16,3 \\
\hline ahpé & 17,3 \\
\hline ahpé & 17,1 \\
\hline Ahp & 15,8 \\
\hline Ahp & 16,5 \\
\hline Ahp & 16,8 \\
\hline Ahpé & 17,6 \\
\hline Ahp & 15,2 \\
\hline Ahp & 15,4 \\
\hline Ahpé & 15,4 \\
\hline ahpé & 14,2 \\
\hline ahpé & 15,9 \\
\hline$\overline{A h}$ & 16,1 \\
\hline ahpé & 16,2 \\
\hline Ahp & 16,9 \\
\hline Ah & 14,6 \\
\hline Ahp & 15,9 \\
\hline ahpé & 16,5 \\
\hline Ahp & 16,6 \\
\hline aHpé & 14,0 \\
\hline$A p$ & 17,3 \\
\hline$\overline{\mathrm{Ah}}$ & 16,8 \\
\hline ahpé & 17,4 \\
\hline Ahp & 17,7 \\
\hline ahp & 14,7 \\
\hline ahpé & 14,6 \\
\hline ahpé & 17,3 \\
\hline ahpé & 15,9 \\
\hline aHpé & 17,1 \\
\hline Ahp & 15,8 \\
\hline ahp & 15,2 \\
\hline aHpé & 17,0 \\
\hline ahpé & 16,3 \\
\hline ahpE & 15,2 \\
\hline ah é & 18,5 \\
\hline Ahp & 14,7 \\
\hline ahp & 15,8 \\
\hline Ahp & 14,9 \\
\hline \multirow[t]{2}{*}{$\mathrm{hp}$} & 14,3 \\
\hline & 16,4 \\
\hline ahpé & 18,0 \\
\hline ahp & 16,2 \\
\hline ahpé & 14,6 \\
\hline Ahp & 18,0 \\
\hline ahp & 14,9 \\
\hline ahp & 15,5 \\
\hline
\end{tabular}

\begin{tabular}{|c|}
\hline Principal \\
\hline Principal \\
\hline Principal \\
\hline Principal \\
\hline Principal \\
\hline Secondaire \\
\hline Principal \\
\hline Principal \\
\hline Principal \\
\hline Principal \\
\hline Principal \\
\hline Principal \\
\hline Principal \\
\hline Secondaire \\
\hline Secondaire \\
\hline Principal \\
\hline Principal \\
\hline Secondaire \\
\hline Principal \\
\hline Principal \\
\hline Principal \\
\hline Principal \\
\hline Principal \\
\hline Secondaire \\
\hline Principal \\
\hline Principal \\
\hline Principal \\
\hline Principal \\
\hline Secondaire \\
\hline Secondaire \\
\hline Principal \\
\hline Secondaire \\
\hline Principal \\
\hline Principal \\
\hline Secondaire \\
\hline Principal \\
\hline Principal \\
\hline Principal \\
\hline Principal \\
\hline Principal \\
\hline Principal \\
\hline Principal \\
\hline Secondaire \\
\hline Principal \\
\hline Secondaire \\
\hline Srincipal \\
\hline Secondaire \\
\hline
\end{tabular}

\begin{tabular}{|l|}
\hline Constante \\
\hline Constante \\
\hline Constante \\
\hline Constante \\
\hline Constante \\
\hline Constante \\
\hline Constante \\
\hline Constante \\
\hline Constante \\
\hline Constante \\
\hline Inconstante \\
\hline Constante \\
\hline Inconstante \\
\hline Constante \\
\hline Inconstante \\
\hline Constante \\
\hline Constante \\
\hline Constante \\
\hline Constante \\
\hline Inconstante \\
\hline Inconstante \\
\hline Constante \\
\hline Inconstante \\
\hline Inconstante \\
\hline Constante \\
\hline Inconstante \\
\hline Constante \\
\hline Constante \\
\hline Inconstante \\
\hline Inconstante \\
\hline Constante \\
\hline Inconstante \\
\hline Constante \\
\hline Inconstante \\
\hline Inconstante \\
\hline Constante \\
\hline Constante \\
\hline Inconstante \\
\hline Constante \\
\hline Inconstante \\
\hline Constante \\
\hline Inconstante \\
\hline Inconstante \\
\hline \\
\hline Inconstante \\
\hline Constante \\
\hline
\end{tabular}

\begin{tabular}{|c|}
\hline Longue \\
\hline Longue \\
\hline Longue \\
\hline Longue \\
\hline Longue \\
\hline Longue \\
\hline Longue \\
\hline Courte \\
\hline Longue \\
\hline Longue \\
\hline Courte \\
\hline Courte \\
\hline Longue \\
\hline Longue \\
\hline Courte \\
\hline Courte \\
\hline Longue \\
\hline Courte \\
\hline Courte \\
\hline Longue \\
\hline Longue \\
\hline Courte \\
\hline Longue \\
\hline Courte \\
\hline Longue \\
\hline Longue \\
\hline Longue \\
\hline Courte \\
\hline Courte \\
\hline Courte \\
\hline Longue \\
\hline Longue \\
\hline Longue \\
\hline Courte \\
\hline Courte \\
\hline Longue \\
\hline Courte \\
\hline Courte \\
\hline Longue \\
\hline Longue \\
\hline Courte \\
\hline Longue \\
\hline Longue \\
\hline Courte \\
\hline Courte \\
\hline
\end{tabular}


MG. La coagulation n'est favorablement influencée par la $\kappa-\mathrm{CN}(P=0,05$ avec temps de floculation, $\mathrm{K} 15$ et $\mathrm{A} 30$ ) et par le calcium ( $P=0,05$ avec le temps de floculation seulement) que dans les laits standardisés. L'influence de l' $\alpha$-CN apparaît constamment non significative.

\section{Paramètres de composition des laits et fromagerie}

Des corrélations très significatives $(P<0,001)$ ont été calculées entre les rendements de fabrication exprimés en $\mathrm{kg}$ de fromage (rendement brut) et en $\mathrm{kg}$ de MAT (rendement MAT) obtenus à partir de 100 I de lait (fig 8) et les paramètres de la coagulation mesurés sur les laits stan-dardisés en MG utilisés.

Comme l'illustre la figure 9 , dans le cas du rendement brut, ces corrélations sont négatives avec l'indice de raffermissement $\mathrm{K} 15$ et positives avec l'indice de fermeté du caillé A30.

Parmi les paramètres de composition étudiés sur les laits standardisés en MG, seules la $\beta-\mathrm{CN}(P<0,001)$, la somme $\alpha-+\beta-+\kappa-\mathrm{CN}$ et le TP $(P=0,05)$ apparaissent corrélés au rendement brut de fabrication. Pour le rende- ment MAT, plus fortement corrélé au TP ( $P$ $<0,001)$ qu'à la $\beta-C N$ et à la somme $\alpha-+\beta$ $+\kappa-\mathrm{CN}(P=0,01)$, il existe aussi une faible corrélation positive avec l' $\alpha-\mathrm{CN}(P=0,1)$ ainsi qu'une corrélation négative avec le TB ( $P$ $=0,05)$ du lait utilisé.

\section{DISCUSSION}

Plusieurs travaux précédemment publiés (Collard-Bovy et al, 1991 ; El Bari et al, 1991 ; 1992 ; Humbert et al, 1991 ; Marchal et al, 1991 ; Montagne et al, 1991 ; Haïssat et al, 1994) ont démontré que l'immunonéphélémétrie microparticulaire était, dans ses applications au lait de vache comme dans celles à la biologie clinique humaine (Montagne et al, 1992a ; 1992b), une méthode d'immunoanalyse à la fois simple, rapide, fiable et précise. Utilisée dans ce travail pour doser les $\alpha-C N, \beta-C N$ et $\kappa-\mathrm{CN}$ dans un nombre important de laits de troupeau et de fromagerie, sa précision y est confirmée par la valeur des coefficients de variation obtenus en répétabilité intra- $(2,9$ à $3,3 \%$ ) et inter-essais $(3,7$ à $7,0 \%)$ sur les 13 mois de l'étude.

L'analyse globale des concentrations des $3 \mathrm{CN}$ obtenues indique une sous-estimation

Fig 7. Caractère favorable (cadre gras) et défavorable (cadre mince) des facteurs qui déterminent, à la production, les paramètres de composition des laits de troupeau. Les troupeaux sont classés selon la somme $\alpha-+\beta-+\kappa-C N$. (Races : $N$ : Normande dominante, $F$ : FFPN dominante, $M$ : troupeau mixte. Sols : TF : très favorable, $\mathrm{F}$ : favorable, $\mathrm{PF}$ : peu favorable, $\mathrm{FS}$ : favorable si drainage. Répartition des vêlages : a : automne, $h$ : hiver, $\mathrm{p}$ : printemps, é : été, la saison principale est indiquée en majuscule. La ration alimentaire journalière est indiquée favorable quand elle est égale ou supérieure à la ration moyenne, $16,2 \mathrm{~kg} / \mathrm{vache} / \mathrm{jour}$. Ensilage de maïs : aliment principal ou secondaire en stabulation. Alimentation complémentaire : constante ou inconstante. La transition stabulation - pâturage est dite longue quand elle est égale ou supérieure à 6 semaines).

Favourable (bold-faced border) and unfavourable (thin border) state of the factors defining the composition parameters in herd milks. Herds are ranged according to the total amount $\alpha-\beta-\beta-\kappa-C N$. (Breed: N: Normande dominant, F: FFPN dominant, M: composite herd. Sol: TF: very favourable, F: favourable, PF: disfavourable, FS: favourable when drained. Distribution of calving: a: autumn, $h$ : winter, p: spring, é: summer, the main season in capital. Daily food intake: marked favourable when $\geq 16.2 \mathrm{~kg} / \mathrm{cow} / \mathrm{jday}$, mean intake. Corn ensilage: principal or secondary feed during stabbling. Complementary feeding: constant or inconstant. Transition stabulation - grazing: long: $\geq 6$ weeks). 


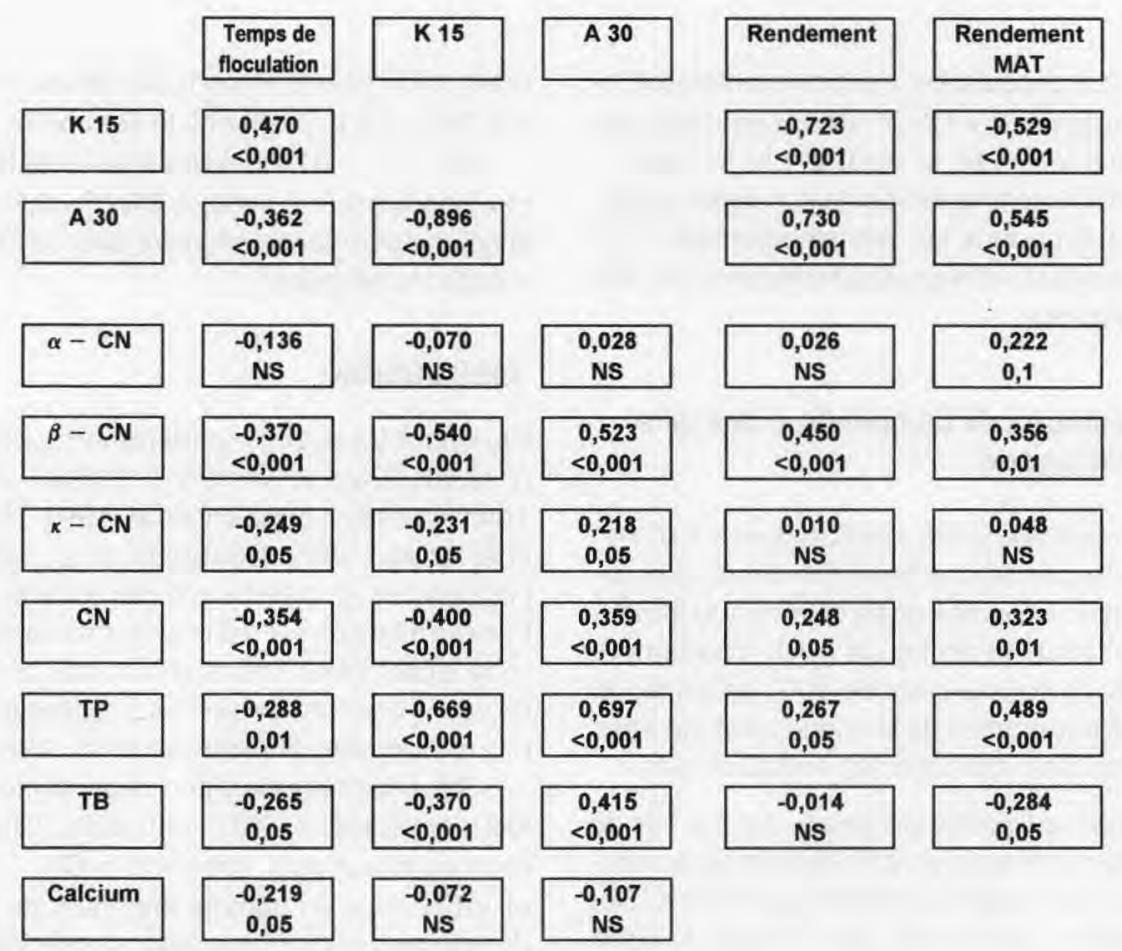

Fig 8. Corrélations (coefficients de corrélation et signification) entre les paramètres mesurés sur l'ensemble des laits de fromagerie standardisés en MG étudiés $(n=89)$, et entre ces paramètres dans les laits utilisés en fabrications fromagères et le rendement de ces fabrications ( $n=65)$. (K15 : indice de raffermissement du caillé ; $\mathrm{A} 30$ : indice de fermeté ; $\mathrm{CN}$ : somme $\alpha-+\beta-+\kappa-\mathrm{CN}$; NS : non significatif à $P<0,1)$.

Correlations (correlation coefficients and signification) between the parameters measured in all fat-standardized dairy-milks ( $\mathrm{n}=89$ ), and between these parameters in the dairy-milks used in cheese-making and the production yield $(\mathrm{n}=65)$. (K15: rheological parameter representing the time required for the curd to achieve a specified firmness; $A 30$ : firmness parameter; $C N$ : total amount $\alpha-+$ $\beta-+\kappa-C N ; N S$ : not significant at $\mathrm{P}<0.1)$.

relative de leurs valeurs, de celle de leur somme et du rapport de cette somme sur le TP, par rapport aux valeurs couramment admises (Swaisgood, 1992). Une étude comparative des résultats obtenus par immunonéphélémétrie microparticulaire avec ceux fournis par d'autres méthodes (électrophorèse, filtration sur gel, échange d'ions, Kjeldahl...) a été précédemment effectuée (Humbert et al, 1991). Elle montre la grande dispersion des résultats obtenus par ces méthodes $(11,2$ à $15,6 \mathrm{~g} / \mathrm{l}$ pour $\alpha-\mathrm{CN}$ et 1,6 à 3,9 g/l pour $\kappa-C N$ par exemple) et indique l'influence, sur les valeurs mesurées, de la technique de dosage mise en oeuvre. La technique employée ici est une méthode immunochimique dont la spécificité est celle de la réaction entre l'antigène (analyte et étalon) et les anticorps d'antisérums dont la réactivité et la spécificité ont été préalablement établies (Collard-Bovy et al, 1991 ; El Bari et al, 1991). Il a été en particulier montré que ces antisérums reconnaissaient préférentiellement les antigènes non dégradés 


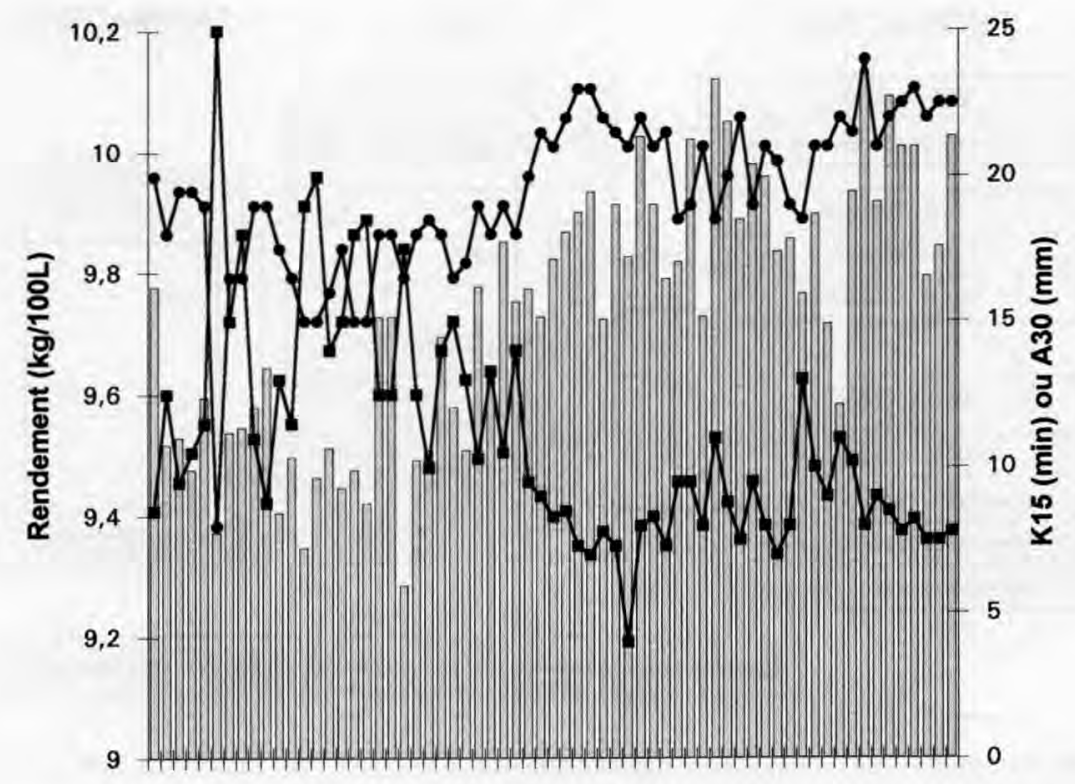

Fabrication

Fig 9. Évolution comparée sur 65 fabrications fromagères industrielles du rendement (histogramme), de l'indice de raffermissement K15 (*) et de l'indice de fermeté A30 $(\bullet)$ mesurés sur les laits de fromagerie standardisés en MG, utilisés pour ces fabrications.

Comparative evolution observed in 65 industrial productions of cheese between yield (histogram), the rheological parameter $K 15$ representing the time required for the curd to achieve a specified firmness $(\bullet)$, and the firmness parameter $A 30(\bullet)$ measured in the fat-standardized dairy-milks used.

(Humbert et al, 1991 ; El Bari et al, 1992). Une poudre de lait, préparée dans des conditions peu dénaturantes, puis étalonnée et validée pour l'immunonéphélémétrie microparticulaire au cours d'une étude (Collard-Bovy et al, 1991) réalisée sur 260 laits entiers frais, est d'autre part utilisée comme standard. En l'absence de référence à un étalon international destiné aux dosages immunochimiques des différentes $\mathrm{CN}$, il n'est évidemment pas possible d'affirmer avec certitude la justesse absolue de cette standardisation. Que celleci soit ou non responsable de la sous-estimation des concentrations des $\alpha$-, $\beta$ - et $\kappa-\mathrm{CN}$ mesurées dans les laits et de la faiblesse du rapport de leur somme sur le TP, l'intérêt de l'analyse des différentes fractions caséiques n'est toutefois pas remis en cause puisque celles-ci sont toutes affectées dans les mêmes proportions et que l'estimation de leur part relative reste donc correcte.

L'étude comparative effectuée sur les concentrations des $\alpha-\mathrm{CN}, \beta-\mathrm{CN}$ et $\kappa-\mathrm{CN}$ mesurées par immunonéphélémétrie microparticulaire et celles des autres paramètres de composition traditionnellement mesurés sur les laits suggère qu'un lait riche en l'une des $3 \mathrm{CN}$ majeures, l'est aussi pour les 2 autres et pour les TP, TB, lactose. $\mathrm{L}^{\prime} \alpha-\mathrm{CN}$, fraction de $\mathrm{CN}$ dominante dans la micelle, apparaît logiquement comme le meilleur marqueur de la valeur de la somme $\alpha-+\beta-+\kappa-C N$, tandis que l'analyse des indices moyens de variation indique que la $\beta$ - $\mathrm{CN}$ apparaît comme le meilleur marqueur de son évolution. 


\section{$\begin{array}{lll}\text { PRODUCTION LAIT } & \text { FROMAGERIE }\end{array}$}

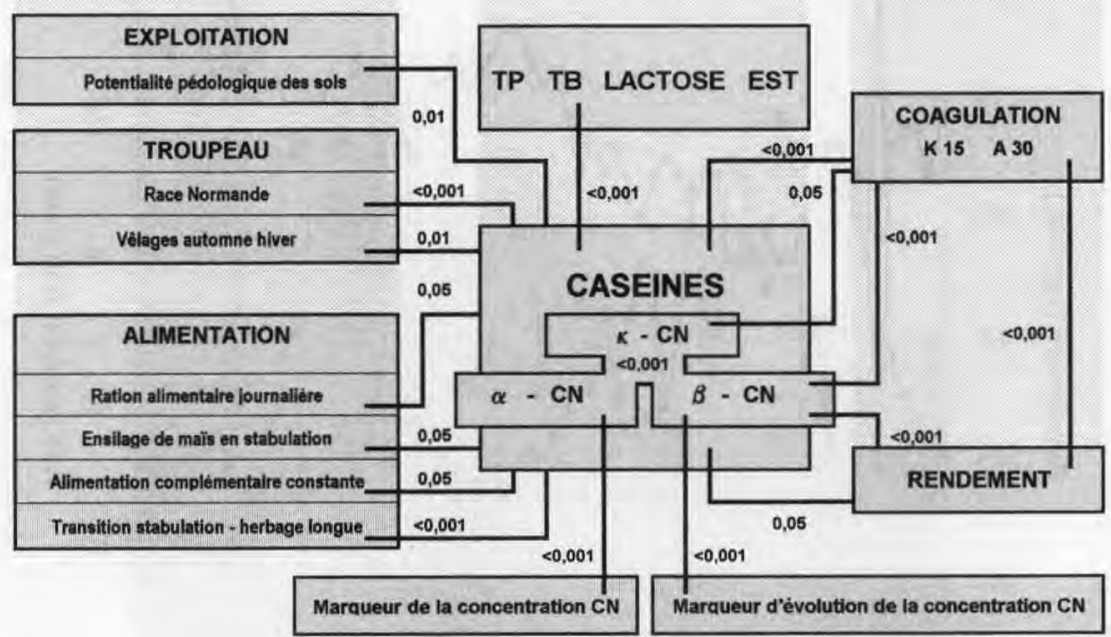

Fig 10. Paramètres de composition biochimique du lait, poids des principaux facteurs qui les déterminent à la production et influence en fromagerie.

Parameters of the biochemical composition of milk, weight of the main factors defining these parameters at production and their influence on cheese-making.

L'analyse des paramètres de composition, traditionnellement mesurés sur les laits, en fonction des facteurs de production, définis par l'étude des variations individuelles non expliquées par les variations moyennes de la somme des $3 \mathrm{CN}$ dosées par immunonéphélémétrie microparticulaire, permet de confirmer l'influence, déjà étudiée en Normandie (Desnouveaux, 1984) et bien connue dans d'autres régions françaises (Mahieu, 1985 ; Coulon et al, 1988 ; Garel et Coulon, 1990 ; Martin et Coulon, 1991), de certains de ces facteurs de production sur la composition du lait et sa qualité fromagère, à savoir i) la race (Storry et al, 1983 ; Vertès et al, 1989 ; Macheboeuf et al, 1993) ; ii) l'alimentation (Rémond, 1985 ; Laurent et Gardeur, 1989 ; Sutton, 1989 ; Vertès et Hoden, 1989 ; Vertès et al, 1989 ; Coulon et Rémond, 1991 ; Laurent et al, 1991 ; Hauwuy et al, 1992) ; iii) les conditions de transition entre stabulation et pâturage (Grandison et al, 1984 ; Vertès et al, 1989; Macheboeuf et al, 1993) ; iv) les in- fluences associées de la saison de production, de la répartition des vêlages, du stade de lactation et des variations climatiques (Chapman et Burnett, 1972 ; Rémond, 1987 ; Rémond et Journet, 1987 ; Agabriel et al, 1990).

II convient d'ajouter à ces facteurs de production, la nature du sol exploité : celle-ci détermine non seulement la qualité de l'herbe (Duchaufour, 1970), aliment de base principal pour la grande majorité des troupeaux pendant 6 à 8 mois de l'année, mais aussi celle des aliments auto-produits tels que le foin ou l'ensilage d'herbe. Cependant, la relation statistique observée dans ce travail entre le type de sol et la composition du lait serait plus facilement interprétable en présence d'une étude de la composition floristique et de la valeur énergétique ou nutritionnelle des herbages.

L'étude individuelle des troupeaux montre que la production d'un lait de qualité apparaît 
plus comme le résultat de l'association, au niveau de l'élevage, de plusieurs facteurs favorables, que de la prééminence de l'un d'entre eux. Cependant, les interactions possibles entre les facteurs qui déterminent les paramètres de composition biochimique des laits de troupeau dans des conditions normales de production, rendent leur étude fort complexe. Ainsi, comme cela a déjà été rapporté pour des troupeaux FFPN et Montbéliards (Coulon et Lilas, 1988), la plus grande fréquence de l'ensilage de maïs comme aliment principal des troupeaux FFPN en stabulation pourrait expliquer au moins en partie le plus faible écart du TB et des $\mathrm{CN}$ en hiver entre troupeaux FFPN et Normands, alors qu'en été seul l'effet génétique favorable des Normandes s'exprimerait.

Le groupe des 50 troupeaux étudiés se veut représentatif de l'ensemble de ceux dont la production est collectée par une laiterie Normande de taille moyenne. Une grande amplitude est ainsi observée dans la distribution de leurs principales caractéristiques (nombre de vaches, quota, surface exploitèe par l'éleveur) et des concentrations des paramètres mesurés dans leur lait. Ces concentrations sont significativement un peu plus élevées que dans les laits de fromagerie crus étudiés. Le lait produit par le groupe de troupeaux, sélectionnés sur des critères de race dominante, de qualité bactériologique et de TP du lait, n'est donc pas totalement représentatif de l'ensemble des laits, collectés par la même laiterie et qui constituent les laits de fromagerie étudiés. Cependant sur les 13 mois du suivi de chaque paramètre de composition, un parallélisme d'évolution des laits de troupeau et des laits de fromagerie crus a pu être constaté. De même, les liens de dépendance statistique observés entre les paramètres dans les laits de troupeau sont retrouvés entre ceux des laits de fromagerie crus. L'étude amont, à la ferme, et l'étude aval, en fromagerie, peuvent donc être raisonnablement réunies dans une étude globale, s'étendant de la production du lait jus- qu'à sa valorisation industrielle par transformation fromagère.

La comparaison entre laits de fromagerie crus et standardisés en MG montre que l'efficacité de cette standardisation ne s'accompagne pas d'une restriction de l'amplitude de variation des $\mathrm{CN}$ et n'altère donc pas les possibilités de mise en évidence des liens statistiques susceptibles d'exister entre elles et les autres paramètres de composition, de coagulation et de fromagerie.

L'aptitude à la coagulation du lait à la présure est largement dépendante de sa composition protéique (Vertès et Hoden, 1989 ; Macheboeuf et al, 1993) et ionique (Okigbo et al, 1985 ; Nakamura et Niki, 1993), mais les paramètres de celle-ci n'ont pas tous le même degré d'influence sur la succession des étapes qui transforment le lait en fromage (Remeuf et al, 1991). Après standardisation du lait en $\mathrm{MG}$, la $\beta-\mathrm{CN}$ et la somme $\alpha-+\beta-+\kappa-C N$ influencent fortement l'ensemble des paramètres de coagulation et semblent favoriser son processus dans toutes ses étapes, tandis que l'effet de la $\mathrm{\kappa}-\mathrm{CN}$ et du calcium apparaît limité aux phases précédant la phase de gélification (Scher, 1988). Le rôle du pH dans l'aptitude du lait à la coagulation (Noël et al, 1991) n'a pas été envisagé ici. L'étude d'un nombre restreint de laits de troupeau indique l'étroitesse des variations de ce paramètre $(n=274, \min =6,66$, $\max =6,96, \operatorname{moy}=6,81, \mathrm{ET}=0,06$ ) et les fabrications fromagères sont effectuées à $\mathrm{pH}$ standardisé.

L'étude de fabrications fromagères industrielles permet de confirmer par ailleurs l'étroite dépendance entre l'aptitude à la coagulation à la présure du lait entrant en fabrication et les rendements de celle-ci (Lawrence et al, 1984). Ces rendements sont cependant fortement influencés et nivelés par les conditions de fabrication (Lenoir et al, 1985), définies par le fromager et qui ne sont pas prises en compte dans cette étude. Parmi les 3 CN majeures, l'influence de la $\beta-C N$ 
apparaît prééminente et l'influence favorable de sa concentration sur la coagulation à la présure (Yun etal, 1982 ; Pearse etal, 1986 ; Remeuf et al, 1989) se retrouve sur les rendements de fabrication. Elle est cependant plus importante sur le rendement brut que sur le rendement MAT et favoriserait principalement la synérèse du caillé et la rétention d'eau lors de son égouttage (Colin et al, 1992). L'intérêt de la mesure par immunonéphélémétrie microparticulaire de la concentration des laits en $\beta-C N$, dans la prédiction du rendement fromager brut et sa prise en compte dans des équations prédictives de ce rendement telles que celles déjà établies (Maubois et al, 1970 ; Colin et al, 1992) pourraient être considérés.

\section{CONCLUSION}

Le cadre du travail rapporté dans cette étude est limité, dans le temps et surtout dans l'espace : quelques kilomètres carrés de Normandie, une laiterie de taille moyenne, une fabrication fromagère particulière. Ses implications sont cependant plus larges.

Notre travail évalue en effet globalement la qualité du lait, de sa production jusqu'à sa valorisation fromagère. La figure 10 propose une synthèse des résultats de cette évaluation : le dosage par immunonéphélémétrie microparticulaire des $\alpha$-CN (marqueur de la valeur de la somme des $3 \mathrm{CN}$ majeures), $\beta$ $\mathrm{CN}$ (marqueur de l'évolution de cette somme) et $\kappa$-CN permet de retrouver des notions bien connues et abondamment publiées en zootechnie (poids, relatif et cumulatif, des principaux facteurs de production, liés à l'exploitation, au troupeau, à sa conduite et à son alimentation) et en technologie fromagère (paramètres permettant de définir au mieux l'aptitude du lait à cette transformation).

Les différents acteurs de la filière lait ont conscience de l'importance de produire et de transformer une matière première de haute qualité. Les moyens traditionnels d'évaluation de cette qualité existent. Ils se complètent aujourd'hui de nouvelles techniques d'immunoanalyse, comme l'immunonéphélémétrie microparticulaire, souvent développées à l'origine pour la biologie humaine et qui conservent dans leurs applications au domaine de l'agro-alimentaire, leurs caractéristiques de spécificité, de sensibilité, de simplicité et de rapidité.

\section{REMERCIEMENTS}

Les auteurs adressent leurs plus vifs remerciements aux 50 éleveurs qui, 2 fois par mois pendant 13 mois, ont accepté de fournir avec un échantillon de leur production, des informations précises et précieuses sur leur exploitation et leur troupeau, ainsi qu'à l'ensemble des personnes qui ont permis la réalisation de l'étude sur les laits de fromagerie et les fabrications fromagères industrielles.

Nous remercions également ARILAIT qui a coordonné un programme de recherche au cours duquel certains protocoles d'analyse utilisés dans cette étude ont été mis au point.

\section{RÉFÉRENCES}

Agabriel C, Coulon JB, Marty G, Cheneau N (1990) Facteurs de variation du taux protéique du lait de vache. Étude dans les exploitations du Puy-de-Dôme. INRA Prod Anim 2, 137-150

Biggs DA, Johnsson G, Sjaunja LO (1987) Analysis of fat, protein, lactose and total solids by infra-red absorption. Int Dairy Fed Bull 208, 21-30

Boquien CY (1993) L'apport de l'immunochimie en agro-alimentaire. Immunoanal Biol Spéc2, 81-88

Chapman HR, Burnett J (1972) Seasonal changes in the physical properties of milk for cheesemaking. Dairy Ind 37, 207-211

Colin O, Laurent, F (1991) Qualité du lait et transformation fromagère. In : Protéines et rendements en industries laitières. Colloque ENSAIA (INPL), Nancy

Colin O, Laurent F, Vignon B (1992) Variations du rendement fromager en pâte molle. Rela- 
tions avec la composition du lait et les paramètres de la coagulation. Lait, 72, 307-319

Collard-Bovy C, Marchal E, Humbert G, Linden G, Montagne P, El Bari N, Duheille J, Varcin P (1991) Microparticle-enhanced nephelometric immunoassay. I- Measurement of Alphas-Casein and Kappa-Casein. J Dairy Sci 74, 3695-3701

Collin JC, Kokelaar A, Rollet-Repecaud O, Delacroix-Buchet A (1991) Dosages des caséines du lait de vache par électrophorèse et par chromatographie liquide rapide d'échanges d'ions (FPLC) : comparaison des résultats. Lait 71, 339-350

Coulon JB (1991) Facteurs de variation du taux protéique du lait de vache en exploitation : réflexions à partir de résultats d'enquêtes. In : Qualité des laits et aptitude fromagère. Colloque INRA-ENSAR, Rennes

Coulon JB, Lilas JP (1988) Composition chimique et contamination butyrique du lait : Facteurs de variations dans le département de la Haute-Loire. INRA Prod Anim 1, 201-207

Coulon JB, Roybin D, Congy E, Garret A (1988) Composition chimique et temps de coagulation du lait de vache : facteurs de variations dans les exploitations du Pays de Thônes. INRA Prod Anim 1, 253-263

Coulon JB, Rémond B (1991) Réponses de la production et de la composition du lait de vache aux variations d'apports nutritifs. INRA Prod Anim 4, 303-309

CPCS (1967) Commission de pédologie et de cartographie des sols. Classification Française des sols. ENSA, Grignon

Desnouveaux R (1984) Contribution à l'étude biochimique d'un lait cru : exemple du bassin d'Isigny-sur-mer. Thèse de Doctorat de troisième cycle, Université de Nancy 1

Doré $F$, Juignet $P$, Larsonneur $C$, Pareyn $C$, Rioult M (1977) Guides géologiques régionaux, Normandie. Masson, Paris

Duchaufour P (1970) Précis de pédologie. Masson, Paris

El Bari N, Montagne P, Humbert G, Cuillière ML, Varcin P, Linden G, Duheille J (1991) Development of a microparticle-enhanced nephelometric immunoassay for the quantification of
Beta-casein in milk. Food Agric Immunol 3, 63-71

El Bari N, Montagne P, Cuillière ML, Humbert G, Linden G, Duheille J (1992) Study of Beta-casein denaturation by microparticle-enhanced nephelometric immunoassay. Food Agric Immunol 4, 229-240

FIL (1981) Fédération internationale de laiterie. Catalogue des fromages, document 141

FIL (1990) Fédération internationale de laiterie. Lait entier. Détermination des teneurs en matière grasse laitière, protéines et lactose. Guide pour l'utilisation des appareils de dosage par absorption dans le moyen infrarouge. Norme internationale provisoire $141 \mathrm{~A}$

Foucart T (1984) Analyse factorielle. Programmation sur micro-ordinateurs, Masson, Paris

Garel JP, Coulon JB (1990) Effet de l'alimentation et de la race des vaches sur la fabrication de fromage d'Auvergne de Saint-Nectaire. INRA Prod Anim 3, 127-136

Goguel J (1967) Carte géologique de la France au $1 / 80000$, Feuille 28 Saint-Lo. BRGM, Orléans

Grandison AS, Ford GD, Owen AJ, Millard D (1984) Chemical composition and coagulating properties of renneted Friesian milk during the transition from winter rations to spring grazing. J Dairy Res 51, 69-78

Grappin R (1992) Bases and experiences of expressing the protein content of milk-France. J Dairy Sci 75, 3221-3227

Grappin R, Ribadeau-Dumas B (1992) Analytical methods for milk proteins. In : Advanced dairy chemistry-1: Proteins (PF Fox, ed). Elsevier, London

Guillou H, Miranda G, Pélissier JP (1987) Analyse quantitative des caséines dans le lait de vache par chromatographie liquide rapide d'échange d'ions (FPLC). Lait 67, 135-148

Haïssat S, Marchal E, Montagne P, Humbert G, Béné MC, Faure G, Linden G (1994) Quantitative characterization of bovine plasminogen binding to caseins. Anal Biochem 222, 472 478

Hauwuy A, Paradis J, Coulon JB (1992) Complémentation énergétique de rations à base de foin pour les vaches laitières. INRA Prod Anim $5,339-346$ 
Hoden A, Garel JP, Coulon JB, Laurent F (1991) Influence de quelques facteurs zootechniques sur l'aptitude fromagère du lait de vache. Synthèse de résultats expérimentaux obtenus à I'INRA. In : Qualité des laits et aptitude fromagère. Colloque INRA-ENSAR, Rennes

Humbert G, Collard-Bovy C, Marchal E, Linden G, Montagne P, Duheille J, Varcin P (1991) Microparticle-enhanced nephelometric immunoassay: III-Application to milk and dairy products. J Dairy Sci 74, 3709-3715

Laurent F, Gardeur JN (1989) Effet de la nature et du niveau d'apport en aliment concentré sur les performances de vaches laitières recevant une ration à base d'ensilage de maïs. Ann Zootech 38, 247-258

Laurent F, Parassin PR, Gardeur JN (1991) Influence de facteurs alimentaires sur l'évolution du taux protéique et la qualité des protéines laitières. In : Protéines et rendements en industries laitières. Colloque ENSAIA (INPL), Nancy

Laville P (1993) Unités de terroir naturel et terroir. Une distinction nécessaire pour redonner plus de cohérence au système d'appellation d'origine. Bull O I V 745-746, 227-251

Lawrence RC, Heap HA, Gilles J (1984) A controlled approach to cheese technology. J Dairy Sci $67,1632-1645$

Lenoir J, Lamberet G, Schmidt JL, Tourneur C (1985) La maîtrise du bioréacteur fromage. Biofutur 12, 23-49

Lenoir J, Schneid N (1987) L'aptitude du lait à la coagulation à la présure. In : Le fromage (A Eck, ed). Lavoisier, Paris

Linden G, Chamba JF (1994) La typicité des fromages : une réalité, un objectif. Sci Aliments $14,577-584$

Macheboeuf D, Coulon JB, D'hour P (1993) Etfect of breed, protein genetic variants and feeding on cow's milk coagulation properties. J Dairy Res 60, 43-54

Mahieu $H$ (1985) Facteurs de variation de la composition du lait. In : Laits et produits laitiers. I. Les laits. De la mamelle à la laiterie (M Luquet, ed). Lavoisier, Paris

Marchal E, Collard-Bovy C, Humbert G, Linden G, Montagne P, Duheille J, Varcin P (1991) Microparticle-enhanced nephelometric immu- noassay: II-Measurement of alpha-lactalbumin and beta-lactoglobulin. J Dairy Sci 74 , 3702-3708

Marchand J, Varcin P, Riochet D, Montagne P, Cuillière ML, Duheille J, Pau B (1992) Synthesis of new hydrophilic microspheres : optimized carriers for microparticle-enhanced nephelometric immunoassays. Biopolymers 32 , 971-980

Martin B, Coulon JB (1991) Aptitude fromagère du lait de vache : influence de l'alimentation. Etude réalisée dans 6 exploitations du Pays de Thônes (Haute-Savoie). INRA Prod Anim 4, 209-217

Maubois JL, Ricordeau G, Mocquot G (1970) Étude des rendements en fromagerie de camemberts et de Saint-Paulin. Lait 50, 351-373

Maubois JL (1987) La standardisation en protéines des laits. Techniques actuelles et perspectives d'évolution. In : Le lait, matière première de l'industrie laitière. INRA-CEPIL Paris, 303-307

Montagne P, Gavriloff C, Humbert G, Cuillière ML, Duheille J, Linden G (1991) Microparticleenhanced nephelometric immunoassay for immunoglobulin $\mathrm{G}$ in cow milk. Lait 71, 493 499

Montagne P, Varcin P, Cuillière ML, Duheille J (1992a) Microparticle-enhanced nephelometric immunoassay with microsphere-antigen conjugates. Bioconjugate Chem 3, 187-193

Montagne $P$, Laroche $P$, Bessou T, Cuillière $M L$, Varcin P, Duheille J (1992b) Measurement of eleven serum proteins by microparticle-enhanced nephelometric ímmunoassay. Eur $J$ Clin Chem Clin Biochem 30, 217-222

Nakamura K, Niki R (1993) Rheological properties of casein micelle gels : the influence of calcium concentration on gelation induced by rennet. Biorheology 30, 207-216

Noël Y, Durier C, Lehembre N, Kobilinsky A (1991) Étude multifactorielle de la coagulation mixte du lait analysée par viscoélasticimétrie. Lait 71, 15-39

Okigbo LM, Richardson GH, Brown RJ, Ernstrom CA (1985) Variations in coagulating properties of milk from individual cows. J Dairy Sci 68 , 822-828 
Pearse MJ, Linklater PM, Hall RJ, Mackinlay AJ (1986) Effect of casein micelle composition and casein dephosphorylation on coagulation and syneresis. J Dairy Res 53, 381-390

Piat C (1986) Pour une stratégie de la qualité dans l'industrie laitière. Les principes de la gestion de la qualité. In : Laits et produits laitiers. 3-Qualité, énergie et tables de composition (M Luquet, ed). Lavoisier, Paris

Remeuf F, Lenoir J, Duby C (1989) Étude des relations entre les caractéristiques physicochimiques des laits de chèvre et leur aptitude à la coagulation par la présure. Lait69, 499-518

Remeuf F, Cossin V, Dervin L, Lenoir J, Tomassone R (1991) Relations entre les caractéristiques physicochimiques des laits et leur aptitude fromagère. Lait 71, 397-421

Rémond B (1985) Influence de l'alimentation sur la composition du lait de vache. 2 . Taux protéique : facteurs généraux. Bull Tech CRZV Theix, INRA 62, 53-67

Rémond B (1987) Influence du stade de lactation et de l'âge sur la composition chimique du lait. In : Le lait, matière première de l'industrie laitière. INRA-CEPIL, Paris

Rémond B, Journet M (1987) Effet de l'alimentation et de la saison sur la composition du lait. In : Le lait, matière première de l'industrie laitière. INRA-CEPIL, Paris

Samarajeewa U, Wei Cl, Huang TS, Marshall MR (1991) Application of immunoassay in the food industry. Crit Rev Food Sci Nutr 29, 403-434

Scher J (1988) Contribution à l'étude de l'influence de la composition du lait et de la taille des micelles sur la coagulation enzymatique: approche statistique et phénoménologique. Thèse INPL, Nancy

Sommer HH, Matsen $H$ (1935) The relation of mastitis to rennet coagulability and strengh of milk. J Dairy Sci, 18, 749-755

Storry JE, Grandison AS, Millard D, Owen AJ, Ford GD (1983) Chemical composition and rennet coagulating properties of milks from different breeds and species of ruminants.

J Dairy Res 50, 215-229

Sutton JD (1989) Altering milk composition by feeding. J Dairy Sci 72, 2801-2814

Swaisgood HE (1992) Chemistry of the caseins. In : Advanced Dairy Chemistry. 1. Proteins (PF Fox, ed). Elsevier, London

Tarodo de la Fuente B, Alais C, Frentz R (1969) Étude de la coagulation du lait et de la synérèse par la méthode thromboélastographique. Lait 49, 400-405

Vertès C, Hoden A (1989) Qualité fromagère des laits de vache en fonction des régimes à base d'herbe. Lait 69, 197-209

Vertès C, Hoden A, Gallard Y (1989) Effet du niveau d'alimentation sur la composition chimique et la qualité fromagère des laits de vaches Holstein et Normandes. Résultats préliminaires. INRA Prod Anim 2, 89-96

Wonnacott TH, Wonnacott RJ (1992) Statistique. $4^{\mathrm{e}}$ édn, Economica, Paris

Yun SE, Ohmiya K, Shimizu S (1982) Role of $\beta$-casein in milk curdling. Agric Biol Chem 46, 443-449 